\title{
A highly conserved binding pocket on PP2A-B56 is required for shugoshin binding and cohesion protection
}

Yumi Ueki ${ }^{1}$, Michael A. Hadders², Melanie Bianca Weisser ${ }^{1}$, Isha Nasa ${ }^{3}$, Tanmay Gupta ${ }^{4}$, Emil PT Hertz¹, Thomas Kruse ${ }^{1}$, Guillermo Montoya ${ }^{1}$, A. Arockia Jeyaprakash, Arminja Kettenbach ${ }^{3}$, Susanne M.A. Lens ${ }^{2}$, Jakob Nilsson ${ }^{1,5}$

1 The Novo Nordisk Foundation Center for Protein Research, Faculty of Health and Medical Science, University of Copenhagen, Denmark

2 Oncode Institute and Center for Molecular Medicine, University Medical Center Utrech, Utrecht University, Utrecht, The Netherlands

${ }^{3}$ Biochemistry and Cell Biology, Geisel School of Medicine at Dartmouth College, Dartmouth, United States of America

${ }^{4}$ Wellcome Center for Cell Biology, University of Edinburgh, Edinburgh, Scotland

${ }^{5}$ Correspondence to: jakob.nilsson@cpr.ku.dk 


\section{Abstract}

The shugoshin proteins are universal protectors of centromeric cohesin during mitosis and meiosis. The binding of human Sgo1 to the PP2A-B56 phosphatase through a coiled coil (CC) region is believed to mediate cohesion protection during mitosis. Here we undertook a structure function analysis of the PP2A-B56-Sgo1 complex, revealing unanticipated aspects of complex formation and function. We establish that a highly conserved pocket of the B56 regulatory subunit is required for Sgo1 binding and cohesion protection. Consistent with this, we show that Sgo1 blocks the binding of PP2A-B56 substrates containing a canonical B56 binding motif. Surprisingly, we identify B56 and Sgo1 mutants that prevent complex formation yet support cohesion protection and normal mitotic progression. This suggests that Sgo1 and PP2A-B56 have cohesion protection activity independently of complex formation. Collectively our work provides important insight into cohesion protection during mitosis. 


\section{Introduction}

The shugoshin proteins (Sgo1 (Sgol1) and Sgo2 (Sgol2) in humans) are conserved protectors of centromeric cohesion by preventing premature release of the cohesin complex (Marston, 2015). The first shugoshin protein was discovered in Drosophila melanogaster through the isolation of a mutant, MEI-S332, that lost cohesion prematurely during meiosis (Kerrebrock et al, 1992; Kerrebrock et al, 1995). Subsequent genetic screens identified the shugoshin proteins in yeast (Katis et al, 2004; Kitajima et al, 2004; Marston et al, 2004). Common to these proteins is the presence of an $\mathrm{N}$-terminal coiled coil (CC) region that binds to B56 regulatory subunits hereby localizing PP2A-B56 to centromeres (Kitajima et al, 2006; Riedel et al, 2006; Tang et al, 2006; Xu et al, 2009). The proposed function of the PP2AB56-Sgo1 complex during meiosis is to dephosphorylate Rec8, hereby preventing Separase cleavage of cohesin (Brar et al, 2006; Ishiguro et al, 2010; Katis et al, 2010; Riedel et al., 2006).

The PP2A-B56 protein phosphatase is a Ser/Thr phosphatase that dephosphorylates numerous substrates to regulate mitosis (Garvanska \& Nilsson, 2020; Nilsson, 2019). PP2AB56 is a trimeric holoenzyme composed of a scaffold subunit (PP2A-A) that connects the B56 subunit with the catalytic subunit (PP2A-C) (Fig. 1A)(Cho \& Xu, 2007; Xu et al, 2006). The B56 subunit of the holoenzyme confers substrate specificity by binding to interactors that target the phosphatase to its substrates. Most B56 interactors bind via a conserved LxxIXE peptide motif that engages a highly conserved pocket on B56 present in all five B56 isoforms (Hertz et al, 2016; Wang et al, 2016; Wang et al, 2020; Wu et al, 2017). A number of important mitotic regulators such as BubR1, Kif4A and RacGAP1 bind to PP2A-B56 through a LxxIxE motif to regulate specific dephosphorylation events. There are five isoforms of B56 (B56 $\alpha, \beta, \gamma, \delta$ and $\varepsilon$ ) that display distinct localization patterns during mitosis (Bastos et al, 2014; Foley et al, 2011; Vallardi et al, 2019).

In human somatic cells, Sgo1 and Sgo2 recruit PP2A-B56 $/$ / and to a lesser extent the other PP2A-B56 isoforms, to the centromere (Meppelink et al, 2015; Vallardi et al., 2019). This protects cohesin complexes by locally antagonizing mitotic kinase activity (Kitajima et al, 2005; McGuinness et al, 2005; Salic et al, 2004). Although Sgo2 has been reported to recruit the bulk of PP2A-B56 $\alpha$ to centromeres, Sgo2 is not needed for cohesion protection (Kitajima et al., 2006; Orth et al, 2011; Tang et al., 2006; Vallardi et al., 2019). In contrast, 
depleting Sgo1 prevents cohesion protection despite having limited effect on PP2A-B56 centromeric levels (Kitajima et al., 2006; Tang et al., 2006; Vallardi et al., 2019). Sgo1 performs cohesion protection through a conserved cohesin binding motif that is absent from Sgo2 (Hara et al, 2014; Liu et al, 2013; Nishiyama et al, 2013). Sgo1 furthermore competes directly with the cohesin release factor WAPL for cohesin binding to prevent WAPL activity (Hara et al., 2014). Two proteins, Sororin and the cohesin subunit SA2, have been proposed to be dephosphorylated by PP2A-B56-Sgo1 to protect cohesin (Hauf et al, 2005; Liu et al., 2013; Nishiyama et al., 2013). Indeed, expressing variants of Sororin and SA2 that cannot be phosphorylated bypass the need for Sgo1 (Liu et al., 2013; Nishiyama et al., 2013). However, there are limited data demonstrating that Sororin and SA2 are directly dephosphorylated at centromeres by the PP2A-B56-Sgo1 complex.

In addition to recruiting PP2A-B56, the shugoshin proteins also recruit the chromosomal passenger complex (CPC) to centromeres through their CC region (Kawashima et al, 2007; Tsukahara et al, 2010; Vanoosthuyse et al, 2007). The shugoshin-dependent localization of CPC to the centromere could also contribute to cohesion protection (Hengeveld et al, 2017). Although the interplay between shugoshin recruitment of PP2A-B56 and the CPC to centromeres is not fully established, recent work suggests that the ability of Sgo1/2 to recruit the CPC and PP2A-B56 are distinct activities (Bonner et al, 2020). These observations underscore the complexity of the $\mathrm{CC}$ region of the shugoshin proteins.

Crystallographic studies have determined the human PP2A-B56 $\gamma$ in complex with a fragment of human Sgo1 comprising residues 51-96, which represents most, but not the entire N-terminal CC domain (Xu et al., 2009). This Sgo1 fragment displays less affinity to PP2A-B56 $\gamma$ than longer $\mathrm{N}$-terminal fragments of Sgo1, but sufficient affinity to efficiently bind to PP2A-B56 $\gamma$ under crystallization conditions using high protein concentrations. The structure revealed that the Sgo1 fragment forms a dimer which engages several residues of the last C-terminal HEAT repeat of B56 $\gamma$ and makes contacts to the PP2A catalytic subunit (Fig. 1A). Although the crystal asymmetric unit shows a 1:1 interaction between Sgo1 peptide strands and PP2A holoenzymes, the Sgo1 peptide strands are arranged into a parallel CC homodimer, where one fragment is related to the other by a two-fold crystallographic symmetry axis (depicted as chain A and Asym in Fig. 1A). This arrangement allows them to interact symmetrically with PP2A enzymes on both sides. Thus, one PP2A- 
B56 $\gamma$ holoenzyme displays interactions with residues from both of the two alpha helices forming one Sgo1 CC region in the crystal, which is again consistent with biochemical experiments showing that dimerization of Sgo1 is required for binding to PP2A-B56 (Tang et al, 1998; Xu et al., 2009). In the PP2A-B56y-Sgo1 structure, the LxxlxE binding pocket of B56 $\gamma$ is fully exposed and indeed the $\mathrm{N}$-terminal region of Sgo1 does not appear to contain any recognizable LxxIxE motif.

These observations raise the possibility that the PP2A-B56-Sgo1 complex can make higher order complexes with LxxIxE containing proteins, which could be important for mitotic cohesion protection. We explored this possibility, which revealed unanticipated aspects of the PP2A-B56-Sgo1 complex important for understanding cohesion protection. 


\section{Results and discussion}

\section{Sgo1 and LxxIxE motifs compete for binding to PP2A-B56}

We first determined if Sgo1 can bind to PP2A-B56 in complex with LxxlxE containing proteins. We generated stable inducible HeLa cell lines that express YFP-tagged B56 $\alpha$ (stable inducible HeLa cell lines used throughout unless indicated) and arrested cells in prometaphase using nocodazole. Mitotic cells were collected by mitotic shake-off and YFPB56 $\alpha$ was purified using a YFP affinity resin. This enriches the entire PP2A-B56 $\alpha$ holoenzyme on the beads (Fig. 1B) and co-purifies LxxIxE containing proteins such as BubR1 and Kif4A (Hertz et al., 2016). We then incubated the purified YFP-B56 $\alpha$ with either recombinantly expressed and purified full-length Sgo1 or an N-terminal fragment of Sgo1 spanning residues 1-154 and washed the complexes (Fig. 1C). As a control, we treated YFP-B56 $\alpha$ purifications with buffer instead of Sgo1. Strikingly, both BubR1 and Kif4a bound to PP2A-B56 $\alpha$ in the control samples but were efficiently displaced in the presence of Sgo1(Fig. 1D). We performed a similar experiment in the presence of a high affinity LxxIxE peptide or the control peptide LxxAxA. The LxxIxE peptide efficiently displaced BubR1 and Kif4A as expected but also reduced Sgo1 binding (Fig. 1E). These results suggest that Sgo1 might engage the conserved LxxIxE binding pocket of B56 $\alpha$ for binding. To further confirm this, we used a panel of B56 $\alpha$ mutants that have mutations in the LxxIxE binding pocket and analyzed their ability to bind Sgo1. YFP-B56 $\alpha$ variants were purified from prometaphase arrested cells and Sgo1 and BubR1 binding was analyzed. Interestingly, all B56 $\alpha$ mutants unable to bind BubR1 failed to co-purify Sgo1 (Fig.1 F). The reason why the LxxIxE peptide does not fully displace Sgo1, in contrast to the B56 mutants, could reflect that the PP2AB56-Sgo1 complex is very stable once formed.

Collectively, these results indicate that LxxIxE motif-containing proteins and Sgo1 compete for a common binding surface on PP2A-B56 $\alpha$.

\section{The LxxIxE binding pocket of PP2A-B56 is required for cohesion protection}

The involvement of the B56 $\alpha$ LxxIxE binding pocket in Sgo1 binding was surprising, given that Sgo1 binds the less conserved C-terminal HEAT repeat of B56 $\gamma$ in the reported structure of the PP2A-B56 $\gamma$-Sgo1 complex (Fig. 2A). To further analyze this, we investigated the B56 $\alpha$ 
R222E LxxIxE pocket mutant in depth for Sgo1 binding and cohesion protection. We compared this to a $B 56 \alpha$ mutant $(B 56 \alpha 5 A)$, in which all residues at the reported structural interface with Sgo1 were mutated (B56 $\alpha$ 5A:Y365A, H377A, Y381A, L384A, M388A) (Fig. 2A). First, we compared the binding of PP2A-B56 $\alpha$ to Sgo1 and LxxIxE containing mitotic regulators. Consistent with the reported structure of the PP2A-B56 $\gamma$-Sgo1 complex, we found that YFP-B56 $\alpha$ 5A bound less Sgo1 while maintaining its interactions with BubR1 and Kif4A (Fig. 2B). In contrast, B56 $\alpha$ R222E (mutation in the LxxIxE binding pocket) lost both binding to Sgo1 and LxxIxE containing proteins. In a reciprocal experiment, cells stably expressing FLAG-tagged B56 $\alpha$ variants were transfected with YFP-Sgo1 and then YFPSgo1 was affinity-purified from mitotic cells. Again, we observed impaired binding to both B56 $\alpha$ R222E and 5A, with the latter mutant retaining more binding to Sgo1 (Fig. 2C). A similar result was obtained using YFP-Sgo2 (Fig. EV1A). These experiments strengthen the conclusion that the LxxIxE binding pocket of B56 is an important binding determinant for the shugoshin proteins.

We next analyzed the ability of the B56 $\alpha$ mutants to support cohesion protection. All B56 isoforms were depleted by RNAi and cells were induced to express RNAi resistant YFP. B56 $\alpha$ variants at endogenous levels (Fig. EV1B). This in our hands did not affect Sgo1 or Sgo2 localization to centromeres (Fig. EV1C-D). Cells were synchronized in prometaphase using nocodazole and chromosome spreads were stained with CREST and DAPI to analyze cohesin integrity. The distance between the two peak intensities of CREST was measured, as premature cohesin removal results in longer distances. Indeed, depleting all B56 subunits increased the distance between centromeres, which was rescued by expressing B56 $\alpha$ wild type (WT) (Fig. 2D-E). As anticipated from the interaction studies, B56 $\alpha$ R222E did not support cohesion protection at all while B56 $\alpha$ A surprisingly did (Fig. 2D-E). To further substantiate this result, we performed live cell imaging of the same conditions. Removing Sgo1 and consequently centromeric cohesin results in prolonged mitotic arrest because of activation of the spindle assembly checkpoint. Similarly, depleting all B56 isoforms resulted in a prolonged arrest which was rescued by YFP-B56 $\alpha$ WT and 5A but not the R222E mutant, thus paralleling the chromosome spread results (Fig. EV1E-G). Consistent with our binding experiments (Fig. 2B-C), only YFP-B56 $\alpha$ WT displayed localization to chromosomes as observed by live cell imaging (Fig. EV1F). We analyzed the YFP-B56 $\alpha$ 5A phenotype 
over a range of expression levels and even low levels of expression rescued the B56 RNAi. These results show that mutating the LxxIxE binding pocket of B56 $\alpha$ abolishes cohesion protection while the reported interface for binding the Sgo1 CC appears less critical for this. To establish that B56 $\alpha$ R222E can assemble an active PP2A holoenzyme capable of cohesion protection, we artificially recruited the B56 $\alpha$ mutants to the centromere by fusing them to the centromere-targeting domain of Cenp $B(C B)$. We then asked if in the absence of Sgo1, these B56 $\alpha$ mutants supported cohesion protection (Fig. EV2A-B for Sgo1 depletion). We performed chromosome spreads and measured the distance between CREST peak intensities. All variants of CB-B56 $\alpha$ rescued the cohesion defect when Sgo1 was depleted, arguing that they form functional PP2A complexes (Fig. 2F-G). The results do not exclude the possibility that binding of a LxxIxE interactor to PP2A-B56 is required for cohesion protection under physiological conditions.

Collectively, our analysis of B56 $\alpha$ R222E shows that this mutant is defective in Sgo1 binding and cohesion protection. At present, we do not know if the defect in cohesion protection is due to loss of binding to Sgo1 and/or a LxxIxE interactor.

\section{Sgo1 mutations affecting PP2A-B56 binding}

We were puzzled by the fact that the B56 $\alpha$ A mutant fully supported cohesion protection despite showing a clear reduction in Sgo1 binding. This suggested that binding of PP2AB56 to Sgo1 might not be strictly required for cohesion protection. To explore this further, we investigated the consequence of mutating the residues in Sgo1 involved in binding the C-terminal region of B56. We generated a Sgo1 mutant (Sgo1 4A) where the four residues (L83, K87, Y90, C94) contacting B56y in the reported structure were mutated to alanine residues (Fig. 3A and Fig. EV2C). As a comparison, we used a previously reported Sgo1 $3 A$ mutant (Y57, N60, K62 to alanine) which contains three mutated residues at the interface with the PP2A catalytic subunit (Xu et al., 2009). The reported interface with the PP2A catalytic subunit involves residues from both alpha helices of the Sgo1 CC region (Fig. 3A). Stably expressed YFP-Sgo1 variants were purified from mitotic cells at two salt concentrations (50 mM and $150 \mathrm{mM} \mathrm{NaCl}$ ) and binding to PP2A-B56 $\alpha$ was determined (Fig. 3B-C). Both Sgo1 4A and $3 \mathrm{~A}$ showed a strong reduction in binding to PP2A-B56 
components and only Sgo1 4A maintained some residual binding at the low salt concentration (Fig. 3B).

To analyze binding in cells, we took two separate approaches. Firstly, we employed an assay where Lacl-GFP fusions of Sgo1 variants are localized to a LacO array on chromosome 1 in U2OS cells, which allows visualization of PP2A-B56 and CPC recruitment (Fig. 3D). Mitotic cells expressing Lacl-GFP fusions of Sgo1 full length protein and 1-130 were stained for PP2A-C or CPC components (Aurora B and Borealin), and signals were quantified and normalized to GFP. Compared to Sgo1 WT, both Sgo1 4A and Sgo1 3A mutants recruited PP2A-C less efficiently (Fig. 3D, F and Fig. EV2 A-E). Consistent with the low salt purifications (Fig. 3B), Sgo1 4A recruited slightly more PP2A-C than Sgo1 3A. In contrast, we observed more subtle variations in recruitment of CPC components in the full length Sgo1 constructs while there was no difference using the Sgo1 1-130 constructs (Fig. 3G, $\mathrm{H}$ and Fig. EV2D, E).

In a second approach, we fused the TurbolD tag to the N-terminus of Sgo1 and following addition of biotin to mitotic cells for 1 hour, we enriched biotinylated proteins under stringent purification conditions (Fig. 3I and Fig.EV 3F). Subsequent analysis of samples by label free quantitative mass spectrometry revealed labelling of PP2A-B56 components as well as CPC components and other centromeric proteins (Fig. EV3G, Table EV1). Using this approach, we compared PP2A-B56 binding between the different Sgo1 variants. This revealed a significant ( $p$-value $<0.05$, log2 fold change $>1$ ) reduction in biotinylation of B56 subunits in both Sgo1 3A and 4A compared to Sgo1 WT (Fig. 3J, Table EV1). Consistent with the LacO array results, we observed a reduction in labeling of CPC components Aurora $B$ and Borealin in Sgo1 3A and 4A compared to Sgo1 WT while INCENP labelling was less affected. In particular, we noted a significantly stronger reduction in Borealin labeling in Sgo1 3A (log2 fold change=3.24, $\mathrm{p}<0.05$ ) compared to Sgo1 WT and Sgo1 4A. This subunit has been reported to bind Sgo1 directly (Tsukahara et al., 2010), and thus might be more sensitive to mutations in Sgo1 that affect CPC recruitment. Consistent with our results it has been reported that Sgo1 $3 \mathrm{~A}$ is less efficient in localizing Ipl1 (Aurora B) in budding yeast (Verzijlbergen et al, 2014). Furthermore, we noted lower levels of Plk1 labeling in Sgo1 3A compared to the other Sgo1 proteins (Table EV1). Plk1 has been shown to regulate MEIS322 and human Sgo1 localization to centromeres but we have not noticed any obvious 
differences in centromere localization between the different Sgo1 variants (Clarke et al, 2005; Tang et al., 2006).

Collectively, we define two Sgo1 mutants showing a strong reduction in PP2A-B56 binding and which also have reduced levels of CPC recruitment capacity.

\section{Impairing PP2A-B56 binding to Sgo1 does not prevent cohesion protection}

To determine the ability of Sgo1 mutants to support cohesion protection, we depleted endogenous Sgo1 and expressed RNAi-resistant Sgo1 mutants. Cells were arrested in mitosis; chromosome spreads were prepared and distances between kinetochore pairs were measured (Fig. 4A-B). Sgo1 depletion resulted in complete loss of cohesion, which was rescued by Sgo1 WT and Sgo1 4A but not by Sgo1 3A. In this experimental setup, we observe protection of cohesin along chromosome arms likely due to overexpression of exogenous Sgo1. The lack of cohesion protection in Sgo1 3A is consistent with data from budding yeast meiosis (Xu et al., 2009). To further substantiate these findings, we performed a live cell analysis of cells complemented with the different Sgo1 variants and monitored mitotic progression (Fig. 4C-E). Sgo1 depletion induced a strong mitotic arrest which was rescued by Sgo1 WT and Sgo1 4A but not by Sgo1 3A, consistent with the chromosome spread results. From the live cell analysis, it was obvious that Sgo1 3A expressing cells did not align chromosomes (Fig. 4D). We analyzed all Sgo1 variants at a similar fluorescent intensity as well as over a range of fluorescent intensities, and this revealed that even low levels of Sgo1 4A expression was sufficient to support Sgo1 function.

Given the fact that Sgo1 4A bound slightly more PP2A-B56 compared to Sgo1 3A, this could be sufficient to make Sgo1 $4 A$ functional if complex formation is required for cohesion protection. If this was the case, we reasoned that a partial depletion of B56 regulatory subunits would impact the function of Sgo1 4A more than Sgo1 WT. To test this, we incorporated a B56 RNAi depletion step in our Sgo1 complementation protocol (Fig. EV4A). This resulted in partial depletion of all B56 regulatory subunits (Fig. EV4B) and cells died faster in mitosis compared to Sgo1 depleted cells (Fig. EV4C). It also increased the mitotic timing of Sgo1 WT complemented cells as expected due to the role of PP2A-B56 in establishing kinetochore-microtubule interactions (Foley et al., 2011). However, Sgo1 4A was not more sensitive than Sgo1 WT to depletion of B56 regulatory subunits and 
progressed slightly faster through mitosis. The median time of Sgo1 4A in this experiment was lower than Sgo1 3A complemented cells with endogenous levels of B56 (112 min Sgo1 4A (Fig. EV4C, Sgo1+B56 RNAi) vs 224 min Sgo1 3A (Fig. 4E, Sgo1 RNAi).

The results of the Sgo1 4A mutant show that the binding to PP2A-B56 can be lowered substantially without affecting Sgo1 function, consistent with our analysis of B56 $\alpha 5 A$.

An important discovery from our work is that the highly conserved LxxIxE binding pocket of B56 subunits is required for Sgo1 and Sgo2 binding and cohesion protection. This was surprising based on the reported structure of the human PP2A-B56 $\gamma$-Sgo1 complex and the fact that the B56 binding region of Sgo1 and Sgo2 lacks a recognizable LxxIxE motif. One possibility is that the solved structure, which used only a short fragment of Sgo1, does not fully recapitulate the PP2A-B56-Sgo1 complex and crucial aspects of the structure are yet to be uncovered. We anticipate that full length Sgo1 binds in a manner that engages the LxxIXE binding pocket of B56 as well as the C-terminal HEAT repeat as reported in the structure. Consistent with this, in vivo cross-linking mass spectrometry identified peptides of Sgo1 cross-linked to residues in close proximity to the LxxIxE binding pocket on B56 (Herzog et al, 2012). Given the strong conservation of the B56 LxxIxE binding pocket, our results explain why Sgo1 co-purifies with all isoforms of B56 (Kitajima et al., 2006). In addition to this, specific sequence elements present in B56 $\alpha$ might further favor Sgo1/2 binding (Vallardi et al., 2019). An implication from our results is that Sgo1 and LxxIxE motifs compete for binding to PP2A-B56 which could regulate dephosphorylation during mitosis.

Our work also raises the question of whether PP2A-B56 and Sgo1 are two independent pathways for cohesion protection or whether they act in the same pathway as anticipated from their binding to each other. Experiments with expression of Sororin or Rec8 mutants that cannot be phosphorylated or artificial recruitment of PP2A to cohesin/centromeres does not discriminate between the two models because it could simply be that enhancing the activity of the PP2A-B56 pathway bypasses the need for the Sgo1 pathway. The only way to establish this is to generate separation of function mutants which we have done here.

The results of B56 $\alpha$ A and Sgo1 4A mutants reveal that a substantial reduction in PP2AB56-Sgo1 complex formation can be tolerated without any impact on cohesion protection. Two interpretations are possible based on these results: i) low levels of PP2A-B56 binding 
to Sgo1 is sufficient or ii) binding of PP2A-B56 to Sgo1 is not required. We tested this by lowering the levels of B56 in Sgo1 4A complemented cells, which favored the later interpretation. Collectively, our results with B56 5A and Sgo1 4A support a two-pathway model for cohesion protection during mitosis, though we cannot exclude that residual complex formation supports function.

In contrast, our analysis of B56 $\alpha$ R222E and Sgo1 3A favor that Sgo1 and PP2A-B56 act in the same pathway to protect cohesion. To make this conclusion requires that the $\mathrm{B} 56 \alpha$ R222E and Sgo1 3A mutants specifically disrupt the PP2A-B56-Sgo1 complex. For B56 $\alpha$ R222E, we know that this is not the case as binding to LxxIxE motifs is also disrupted. Our analysis of Sgo1 3A also reveals other changes in the proximity assays, arguing that more work is required to fully establish that the only defect in Sgo1 3A is a loss of PP2A-B56 binding, or cohesion loss in this mutant could be partially attributed to loss of other regulators.

Another Sgo1 mutant that has been analyzed previously is Sgo1 N61 that was originally identified in MEl-S322 (Tang et al., 1998). This mutation prevents PP2A-B56 binding and results in a defect in cohesion protection (Tang et al., 2006; Xu et al., 2009). However, the N61 mutation causes a destabilization of MEI-S322 and this residue is not making any contact to PP2A-B56 in the reported structure (Tang et al., 1998; Xu et al., 2009). It could be that Sgo1 N61 mutation affects the integrity of the CC region which causes the cohesion defect independently of affecting PP2A-B56. Indeed, the MEI-S322 ${ }^{8}$ mutation (V35E in the CC) does not affect B56 subunit interactions yet is compromised in cohesion protection arguing for additional functions of the CC region beyond PP2A-B56 binding (Pinto \& OrrWeaver, 2017; Tang et al., 1998).

Collectively, our work provides important insight into the protection of cohesion during mitosis and pinpoints important questions that needs to be addressed in future studies. 


\section{Acknowledgements}

Work at the Novo Nordisk Foundation Center for Protein Research is supported by grant NNF14CC0001 and JNI is supported by grants from the Danish Cancer Society (R167A10951-17-S2), Independent Research Fund Denmark (8021-00101B) and Novo Nordisk Foundation (NNF180C0053124). This work was also supported by the cryo- EM (NNF0024386) and cryoNET (NNF17SA0030214) grants to G.M. G.M. is a member of the Integrative Structural Biology Cluster (ISBUC) at the University of Copenhagen. M.W. was supported by the Swiss National Fund (P2EZP3_178624) and the Danish Lundbeckfonden (2017-3212). ANK was supported by grants from NIH/NIGMS (R35GM119455). The Orbitrap Fusion Tribrid mass spectrometer was acquired with support from NIH (S10OD016212). MH and SMAL are part of the Oncode Institute, which is partly financed by the Dutch Cancer Society and work for this project was funded by a grant from the Dutch Cancer Society (KWF research project 10366). AAJ acknowledges the Wellcome Trust for their support through the Research Career Development (095822) and Senior Research Fellowship (202811) awards. TG acknowledges the Darwin Trust of Edinburgh for the student ship. We thank Hongtao Yu for providing Sgo1 antibody and Sally Wheatley for providing the Borealin antibody. We thank Adele Marston for comments on the manuscript and fruitful discussions.

\section{Conflict of interest}

GM is a co-founder and board member of Twelve Bio

The rest of the authors declare that they have no conflict of interest 


\section{Figure Legends}

\section{Figure 1. Sgo1 and LxxIxE motifs compete for binding to PP2A-B56}

(A) Structure of the PP2A-B56 $\gamma$-Sgo1 complex (adapted from Xu et al, PDB: 3FGA). The Sgo1 coiled-coil homodimer interacts with both PP2A catalytic and B56 regulatory subunits. The model shows a LxxIxE peptide bound to B56 at its conserved binding pocket. (B) YFP pull down from cells stably expressing YFP (control) or YFP-B56 $\alpha$ enriches the entire PP2AB56 $\alpha$ holoenzyme on the beads. PP2A-A, scaffold subunit; PP2A-C, PP2A catalytic subunit. (C) Coomassie-stained SDS-PAGE of the purified Sgo1 full length (FL) and Sgo1 ${ }^{1-154}$. (D) Competition assay with the purified Sgo1 proteins shown in (C). Binding of YFP-B56 $\alpha$ to indicated proteins was determined. Representative of 3 independent experiments. (E) Peptide competition assay with a WT LxxIXE peptide or a mutated variant that does not bind B56 (LxxAxA). Binding of YFP-B56 $\alpha$ to indicated proteins was determined and quantified by LiCor. (F) YFP-B56 $\alpha$ pull down from cells stably expressing the indicated LxxIxE binding pocket variants of $\mathrm{B} 56 \alpha$ and subsequent immunoblotting of indicated proteins. Representative of 4 independent experiments.

Figure 2. Sgo1 binding to the LxxIxE binding pocket of PP2A-B56 is required for cohesion protection

(A) Structure of the reported PP2A $\gamma$-B56-Sgo1 binding interface and residues mutated in the B56 $\alpha$ 5A mutant are shown. (B) IP of YFP-B56 $\alpha$ from cells stably expressing the B56 $\alpha$ WT, R222E, and 5A followed by immunoblotting of indicated proteins. (C) Reciprocal IP of (B). YFP-Sgo1 expression construct was transfected into cells stably expressing FLAG-B56 $\alpha$ WT, R222E and 5A, followed by YFP IP and immunoblotting of indicated proteins. (D) Representative images of chromosome spreads from the indicated conditions. E) Quantification of (D). The distance between the two peak intensities of CREST was measured for 5 kinetochore pairs and averaged for a single cell and plotted. The data are from 4 independent experiments and the mean and SD are indicated. (F) Sgo1 RNAi and rescue with the indicated B56 $\alpha$ variants fused to YFP and the Cenp B centromere-targeting domain (CB). Representative images of chromosome spreads are shown. CB targets all the rescue constructs (green) to the centromere. (G) Quantification of (F). The distance between 
the two peak intensities of YFP was measured for 5 kinetochore pairs and averaged for a single cell and plotted. The data are from 3 independent experiments and the mean and SD are indicated.

\section{Figure 3. Sgo1 mutations in the coiled-coil domain affect PP2A-B56 binding}

(A) Structure of the reported PP2A-B56 $\gamma-S g o 1$ binding interfaces and residues mutated in the Sgo1 3A and 4A mutants are shown. 3A refers to Y57A, N60A, and K62A mutations at the PP2A-C binding interface. 4A refers to L83A, K87A, Y90A, and C94A mutations at the B56 binding interface of Sgo1. (B-C) IP of YFP-Sgo1 from cells stably expressing the Sgo1 WT, 3A, and 4A using different salt conditions (50mM NaCl (B) or $150 \mathrm{mM}(\mathrm{C})$ ) followed by immunoblotting of indicated proteins and quantification by LiCor. (D-H) Mitotic U-2 OS LacO Haspin CM cells expressing Sgo1-Lacl-GFP variants or Lacl-GFP (control) were stained for PP2A-C (D) or CPC components, Aurora B (E) and Borealin (not shown). PP2A-C (F), Aurora B $(\mathrm{G})$ and Borealin $(\mathrm{H})$ signal intensity was quantified, normalized to GFP, and then plotted. Each circle represents an individual cell, and the mean fluorescent intensity is indicated. Representative of at least 3 independent experiments. (I) Schematic of the TurbolD-Sgo1 approach. (J) Table summarizing the Log2 differences between Sgo1 WT, $3 A$ and $4 A$.

\section{Figure 4. Impaired PP2A-B56 binding to Sgo1 does not prevent cohesion protection}

(A) Representative images of chromosome spreads from Sgo1 RNAi treated cells stably expressing the indicated YFP-Sgo1 variants. All the YFP-Sgo1 rescue constructs (green) localize to the centromeres (CREST, red). (B) The distance between the two peak intensities of CREST was measured for 5 kinetochore pairs from the chromosome spreads in $(A)$ and averaged for a single cell. The data are from 3 independent experiments and the mean and SD are indicated. (C) Experimental protocol of the live cell imaging shown in (D). (D) Sgo1 RNAi and rescue with the indicated Sgo1 RNAi-resistant constructs was performed. Representative still images captured during the live cell imaging showing DIC and YFPSgo1 WT, 3A, and 4A localization during mitosis. Time (min) from nuclear envelop breakdown (NEBD) is indicated. (E) The time from NEBD to anaphase was measured from 
3 independent live cell imaging experiments. Each circle represents an individual cell, and the median is indicated.

\section{Figure EV1.}

(A) YFP IP from cells stably expressing YFP-Sgo2 and transfected with FLAG-B56 $\alpha$ constructs. (B) Validation of the B56 RNAi and rescue system. Endogenous B56 $\alpha$ was efficiently depleted $48 \mathrm{~h}$ after the RNAi treatment. The RNAi resistant YFP-B56 $\alpha$ rescue constructs were expressed approximately at the endogenous level. (C-D) Localization of Sgo1(C) and Sgo2 (D) in cells depleted of B56 and expressing the indicated B56 $\alpha$ variants. Representative images from 3 independent experiments are shown. (E) Experimental protocol of the live cell imaging shown in (F). (F) B56 RNAi and rescue with the indicated B56 $\alpha$ RNAi-resistant constructs were performed. Time (min) from nuclear envelop breakdown (NEBD) is indicated. (G) The time from NEBD to anaphase was measured from 2 independent live cell imaging experiments. Each circle represents an individual cell. Blue circles indicate the cells that were still arrested at the end of filming, and red circles indicate the cells that died during mitosis. The median is indicated with the red horizontal bars.

\section{Figure EV2.}

(A) Validation of the Sgo1 antibody and the Sgo1 RNAi by immunoblotting. While the Sgo1 antibody detects unspecific bands in the whole cell lysates (see input), it is specific for Sgo1 after B56 IP, as the treatment with Sgo1 RNAi completely abolishes Sgo1 signal after 48h. (B) Validation of the Sgo1 antibody and the Sgo1 RNAi by immunofluorescence. Representative immunofluorescent images are shown. (C) The conservation of the Sgo1 coiled-coil region. The residues mutated in Sgo1 3A and 4A are indicated.

\section{Figure EV3.}

(A-E) Mitotic U-2 OS LacO cells expressing Sgo11-130-Lacl-GFP variants or Lacl-GFP (control) were stained for PP2A-C (A) or CPC components, Aurora B (B) and Borealin (not shown). PP2A-C (C), AuroraB (D) and Borealin (E) signal intensity was quantified, normalized to GFP, and plotted. Each circle represents an individual cell, and the mean is indicated. Representative of at least 3 experiments. (F) Blot of stable, doxycycline inducible 
TurbolD-Sgo1 cells treated with doxyxyxline and/or biotin as indicated, and probed for Sgo1 or Streptavidin. (G) Volcano plot of TurbolD-Sgo1 WT cells treated or untreated with biotin. B56 regulatory subunits (2A5A-E) and centromere as well as kinetochore proteins indicated.

\section{Figure EV4.}

(A) Experimental protocol of the live cell imaging with Sgo1 complementation with and without partial B56 depletion shown in (C). (B) WB showing the partial KD of all B56 isoforms. (C) Sgo \pm partial B56 KD and rescue with the indicated Sgo1 RNAi-resistant constructs were performed. The time from nuclear envelop breakdown (NEBD) to anaphase was measured from the live cell imaging. Each circle represents an individual cell. Blue circles indicate the cells that were still arrested at the end of filming, and red circles indicate the cells that died. The median is indicated with the red horizontal bars. Representative of 2 independent experiments.

\section{Table EV1}

Mass spectrometry analysis of TurbolD-Sgo1 samples. 


\section{References}

Bastos RN, Cundell MJ, Barr FA (2014) KIF4A and PP2A-B56 form a spatially restricted feedback loop opposing Aurora B at the anaphase central spindle. J Cell Biol 207: 683693

Berger I, Fitzgerald DJ, Richmond TJ (2004) Baculovirus expression system for heterologous multiprotein complexes. Nat Biotechnol 22: 1583-1587 Bieniossek C, Imasaki T, Takagi Y, Berger I (2012) MultiBac: expanding the research toolbox for multiprotein complexes. Trends Biochem Sci 37: 49-57

Bonner MK, Haase J, Saunders H, Gupta H, Li BI, Kelly AE (2020) The Borealin dimerization domain interacts with Sgo1 to drive Aurora B-mediated spindle assembly. Mol Biol Cell 31: 2207-2218

Branon TC, Bosch JA, Sanchez AD, Udeshi ND, Svinkina T, Carr SA, Feldman JL, Perrimon N, Ting AY (2018) Efficient proximity labeling in living cells and organisms with TurbolD. Nat Biotechnol 36: 880-887

Brar GA, Kiburz BM, Zhang Y, Kim JE, White F, Amon A (2006) Rec8 phosphorylation and recombination promote the step-wise loss of cohesins in meiosis. Nature 441: 532-536 Cho US, Xu W (2007) Crystal structure of a protein phosphatase 2A heterotrimeric holoenzyme. Nature 445: 53-57

Clarke AS, Tang TT, Ooi DL, Orr-Weaver TL (2005) POLO kinase regulates the Drosophila centromere cohesion protein MEI-S332. Dev Cell 8: 53-64 Elias JE, Gygi SP (2007) Target-decoy search strategy for increased confidence in largescale protein identifications by mass spectrometry. Nat Methods 4: 207-214 Eng JK, Jahan TA, Hoopmann MR (2013) Comet: an open-source MS/MS sequence database search tool. Proteomics 13: 22-24

Foley EA, Maldonado M, Kapoor TM (2011) Formation of stable attachments between kinetochores and microtubules depends on the B56-PP2A phosphatase. Nat Cell Biol 13: 1265-1271

Garvanska DH, Nilsson J (2020) Specificity determinants of phosphoprotein phosphatases controlling kinetochore functions. Essays Biochem 64: 325-336 
Hadders MA, Hindriksen S, Truong MA, Mhaskar AN, Wopken JP, Vromans MJM, Lens SMA (2020) Untangling the contribution of Haspin and Bub1 to Aurora B function during mitosis. J Cell Biol 219

Hara K, Zheng G, Qu Q, Liu H, Ouyang Z, Chen Z, Tomchick DR, Yu H (2014) Structure of cohesin subcomplex pinpoints direct shugoshin-Wapl antagonism in centromeric cohesion. Nat Struct Mol Biol 21: 864-870

Hauf S, Roitinger E, Koch B, Dittrich CM, Mechtler K, Peters JM (2005) Dissociation of cohesin from chromosome arms and loss of arm cohesion during early mitosis depends on phosphorylation of SA2. PLoS Biol 3: e69

Hengeveld RCC, Vromans MJM, Vleugel M, Hadders MA, Lens SMA (2017) Inner centromere localization of the CPC maintains centromere cohesion and allows mitotic checkpoint silencing. Nat Commun 8: 15542

Hertz EPT, Kruse T, Davey NE, Lopez-Mendez B, Sigurethsson JO, Montoya G, Olsen JV, Nilsson J (2016) A Conserved Motif Provides Binding Specificity to the PP2A-B56 Phosphatase. Mol Cell 63: 686-695

Herzog F, Kahraman A, Boehringer D, Mak R, Bracher A, Walzthoeni T, Leitner A, Beck M, Hartl FU, Ban N et al (2012) Structural probing of a protein phosphatase 2A network by chemical cross-linking and mass spectrometry. Science 337: 1348-1352 Ishiguro T, Tanaka K, Sakuno T, Watanabe Y (2010) Shugoshin-PP2A counteracts casein-kinase-1-dependent cleavage of Rec8 by separase. Nat Cell Biol 12: 500-506 Katis VL, Galova M, Rabitsch KP, Gregan J, Nasmyth K (2004) Maintenance of cohesin at centromeres after meiosis I in budding yeast requires a kinetochore-associated protein related to MEI-S332. Curr Biol 14: 560-572

Katis VL, Lipp JJ, Imre R, Bogdanova A, Okaz E, Habermann B, Mechtler K, Nasmyth K, Zachariae W (2010) Rec8 phosphorylation by casein kinase 1 and Cdc7-Dbf4 kinase regulates cohesin cleavage by separase during meiosis. Dev Cell 18: 397-409 Kawashima SA, Tsukahara T, Langegger M, Hauf S, Kitajima TS, Watanabe Y (2007) Shugoshin enables tension-generating attachment of kinetochores by loading Aurora to centromeres. Genes Dev 21: 420-435 
Kerrebrock AW, Miyazaki WY, Birnby D, Orr-Weaver TL (1992) The Drosophila mei-S332 gene promotes sister-chromatid cohesion in meiosis following kinetochore differentiation. Genetics 130: 827-841

Kerrebrock AW, Moore DP, Wu JS, Orr-Weaver TL (1995) Mei-S332, a Drosophila protein required for sister-chromatid cohesion, can localize to meiotic centromere regions. Cell 83 : 247-256

Kitajima TS, Hauf S, Ohsugi M, Yamamoto T, Watanabe Y (2005) Human Bub1 defines the persistent cohesion site along the mitotic chromosome by affecting Shugoshin localization. Curr Biol 15: 353-359

Kitajima TS, Kawashima SA, Watanabe Y (2004) The conserved kinetochore protein shugoshin protects centromeric cohesion during meiosis. Nature 427: 510-517 Kitajima TS, Sakuno T, Ishiguro K, lemura S, Natsume T, Kawashima SA, Watanabe Y (2006) Shugoshin collaborates with protein phosphatase 2A to protect cohesin. Nature 441: $46-52$

Kruse T, Gnosa SP, Nasa I, Garvanska DH, Hein JB, Nguyen H, Samsoe-Petersen J, Lopez-Mendez B, Hertz EPT, Schwarz J et al (2020) Mechanisms of site-specific dephosphorylation and kinase opposition imposed by PP2A regulatory subunits. EMBO J 39: e103695

Liu H, Rankin S, Yu H (2013) Phosphorylation-enabled binding of SGO1-PP2A to cohesin protects sororin and centromeric cohesion during mitosis. Nat Cell Biol 15: 40-49 Marston AL (2015) Shugoshins: tension-sensitive pericentromeric adaptors safeguarding chromosome segregation. Mol Cell Biol 35: 634-648

Marston AL, Tham WH, Shah H, Amon A (2004) A genome-wide screen identifies genes required for centromeric cohesion. Science 303: 1367-1370

McGuinness BE, Hirota T, Kudo NR, Peters JM, Nasmyth K (2005) Shugoshin prevents dissociation of cohesin from centromeres during mitosis in vertebrate cells. PLoS Biol 3 : e86

Meppelink A, Kabeche L, Vromans MJ, Compton DA, Lens SM (2015) Shugoshin-1 balances Aurora B kinase activity via PP2A to promote chromosome bi-orientation. Cell Rep 11: 508-515 
Nilsson J (2019) Protein phosphatases in the regulation of mitosis. J Cell Biol 218: 395409

Nishiyama T, Sykora MM, Huis in 't Veld PJ, Mechtler K, Peters JM (2013) Aurora B and Cdk1 mediate Wapl activation and release of acetylated cohesin from chromosomes by phosphorylating Sororin. Proc Natl Acad Sci U S A 110: 13404-13409

Orth M, Mayer B, Rehm K, Rothweiler U, Heidmann D, Holak TA, Stemmann O (2011) Shugoshin is a Mad1/Cdc20-like interactor of Mad2. EMBO J 30: 2868-2880 Pinto BS, Orr-Weaver TL (2017) Drosophila protein phosphatases 2A B' Wdb and Wrd regulate meiotic centromere localization and function of the MEI-S332 Shugoshin. Proc Natl Acad Sci U S A 114: 12988-12993

Riedel CG, Katis VL, Katou Y, Mori S, Itoh T, Helmhart W, Galova M, Petronczki M, Gregan J, Cetin B et al (2006) Protein phosphatase 2A protects centromeric sister chromatid cohesion during meiosis I. Nature 441: 53-61

Salic A, Waters JC, Mitchison TJ (2004) Vertebrate shugoshin links sister centromere cohesion and kinetochore microtubule stability in mitosis. Cell 118: 567-578

Schwanhausser B, Busse D, Li N, Dittmar G, Schuchhardt J, Wolf J, Chen W, Selbach M (2011) Global quantification of mammalian gene expression control. Nature 473: 337-342 Tang TT, Bickel SE, Young LM, Orr-Weaver TL (1998) Maintenance of sister-chromatid cohesion at the centromere by the Drosophila MEI-S332 protein. Genes Dev 12: 38433856

Tang Z, Shu H, Qi W, Mahmood NA, Mumby MC, Yu H (2006) PP2A is required for centromeric localization of Sgo1 and proper chromosome segregation. Dev Cell 10: 575585

Tsukahara T, Tanno Y, Watanabe Y (2010) Phosphorylation of the CPC by Cdk1 promotes chromosome bi-orientation. Nature 467: 719-723

Tyanova S, Temu T, Sinitcyn P, Carlson A, Hein MY, Geiger T, Mann M, Cox J (2016) The Perseus computational platform for comprehensive analysis of (prote)omics data. Nat Methods 13: 731-740

Vallardi G, Allan LA, Crozier L, Saurin AT (2019) Division of labour between PP2A-B56 isoforms at the centromere and kinetochore. Elife 8 
Valot B, Langella O, Nano E, Zivy M (2011) MassChroQ: a versatile tool for mass spectrometry quantification. Proteomics 11: 3572-3577

Vanoosthuyse V, Prykhozhij S, Hardwick KG (2007) Shugoshin 2 regulates localization of the chromosomal passenger proteins in fission yeast mitosis. Mol Biol Cell 18: 1657-1669 Verzijlbergen KF, Nerusheva OO, Kelly D, Kerr A, Clift D, de Lima Alves F, Rappsilber J, Marston AL (2014) Shugoshin biases chromosomes for biorientation through condensin recruitment to the pericentromere. Elife 3: e01374

Wang X, Bajaj R, Bollen M, Peti W, Page R (2016) Expanding the PP2A Interactome by Defining a B56-Specific SLiM. Structure 24: 2174-2181

Wang X, Garvanska DH, Nasa I, Ueki Y, Zhang G, Kettenbach AN, Peti W, Nilsson J, Page R (2020) A dynamic charge-charge interaction modulates PP2A:B56 substrate recruitment. Elife 9

Wu CG, Chen H, Guo F, Yadav VK, Mcllwain SJ, Rowse M, Choudhary A, Lin Z, Li Y, Gu T et al (2017) PP2A-B' holoenzyme substrate recognition, regulation and role in cytokinesis. Cell Discov 3: 17027

Xu Y, Xing Y, Chen Y, Chao Y, Lin Z, Fan E, Yu JW, Strack S, Jeffrey PD, Shi Y (2006) Structure of the protein phosphatase 2A holoenzyme. Cell 127: 1239-1251

Xu Z, Cetin B, Anger M, Cho US, Helmhart W, Nasmyth K, Xu W (2009) Structure and function of the PP2A-shugoshin interaction. Mol Cell 35: 426-441 


\section{Materials and Methods}

\section{Antibodies and RNAi oligos}

Antibodies used in this study were as follows: Rabbit anti-Sgo1 (gift from Dr. Hongtao Yu, 1:200 IF), rabbit anti-Sgo1 (generated in-house, 1:2000 WB), mouse anti-B56a (BD Biosciences 610615, 1:2000 WB and 1:200 IF), rabbit anti-GFP (generated in-house, 1:10,000 WB and 1:500 IF), mouse anti-GFP (Roche \#11814460001, 1:2000 WB and 1:200 IF), mouse anti-BubR1(generated in-house, 1:1000 WB), rabbit anti-Kif4a (Bethyl Laboratories A301-074A, 1:3000 WT), mouse anti-PP2A-C (Millipore clone 1D6 05-421, 1:1000 WB and IF), mouse anti-FLAG M2 (Sigma F3165, 1:10,000 WT), human anti-CREST (Antibodies Inc, 1:500 IF), mouse anti-Aurora B (BD Transductions 611083, 1:1000 IF), rabbit anti-Borealin (gift from Dr. Sally Wheatley), and GFP-Booster Atto488 (Chromotek gba488-100, 1:1000 IF).

RNAi oligos used in this study were: B56 $\alpha$ (Dharmacon 5525), B56 (Dharmacon 5527), B568 (Dharmacon 5528), B56ع (Dharmacon 5529), and Sgo1 (Scilencer Selecct siRNA s45600, Thermo Fischer Scientific).

\section{Cloning}

Standard cloning methods were used throughout the study. pcDNA5/FRT/TO vector was used unless otherwise stated. B56 $\alpha$ variants were generated in our previous study(Hertz et al., 2016). B56 $\alpha$ A, Sgo1 3A, and Sgo1 4A mutant constructs were synthesized by GeneArt (Thermo Fischer Scientific). BamHI and Notl were used to subclone B56 $\alpha$ and Sgo1 constructs with various tags (YFP, FLAG, or TurbolD). Full-length Sgo2 was amplified by $\mathrm{PCR}$ and inserted in pcDNA5/FRT/TO-YFP vector. For YFP-CenpB-B56 $\alpha$ constructs, CenpB domain was amplified by PCR and inserted into pcDNA5/FRT/TO-YFP vector by standard restriction cloning, followed by subcloning of B56 $\alpha$ variants into the vector using BamHI/Notl.

\section{Cell Culture}


HeLa FRT/TRex cells (gift from S. Taylor) were used throughout the study, unless otherwise stated. Stable cell lines were generated using the T-Rex doxycycline Flip-In system (Invitrogen). For synchronization, $2.5 \mathrm{mM}$ thymidine and $200 \mathrm{ng} / \mathrm{uL}$ nocodazole were used.

\section{Expression and purification of recombinant hSgo1}

BL21 (DE3) Gold E.coli cells expressing hSgo1 FL and truncations (hSgo1 ${ }^{1-154}$ ) were grown at $37^{\circ} \mathrm{C} / 200 \mathrm{rpm}$ to an optical density of 1.5 (OD600) and induced overnight at $18^{\circ} \mathrm{C}$ with $0.35 \mathrm{mM}$ IPTG. Cells were resuspended in lysis buffer containing $20 \mathrm{mM}$ Tris. $\mathrm{HCl} \mathrm{pH} 8,500$ $\mathrm{mM} \mathrm{NaCl}, 1 \mathrm{mM}$ EDTA and supplemented with complete EDTA-free cocktail tablets (1 tablet/50ml cells; Roche) and 0.01mg/ml DNase (Sigma) and 1mM PMSF. The lysate was sonicated at $60 \%$ amplitude for 8 minutes (2s on, 2s off) and centrifuged at approx. 58000 $\mathrm{x} \mathrm{g}$ for 50 minutes at $4^{\circ} \mathrm{C}$ and the protein was batch purified using chitin beads (NEB). Post lysis and high salt chaperone wash, the chitin beads were washed with $3 \mathrm{CV}$ of $20 \mathrm{mM}$ Tris. $\mathrm{HCl}, 500 \mathrm{mM} \mathrm{NaCl}, 50 \mathrm{mM}$ DTT and incubated at RT overnight. The next day, the protein was eluted with the lysis buffer without DTT. The elutions were analysed for protein quality on an SDS-PAGE, and the elutions containing hSgo1 were pooled and dialysed in $20 \mathrm{mM}$ Tris $\mathrm{HCl} \mathrm{pH} 8,125 \mathrm{mM} \mathrm{NaCl}, 4 \mathrm{mM}$ DTT overnight at $4^{\circ} \mathrm{C}$. The next day, the dialysed sample was loaded onto a HiTrap Q HP (GE Healthcare) anionic exchange column. The excess DNA contamination was separated from $\mathrm{hSgo1}$ by providing a $50 \%$ salt gradient over $20 \mathrm{CV}$ in an ÄKTA start system (GE Healthcare). The samples containing hSgo1 were pooled, concentrated and the pure protein was finally obtained by a final size exclusion chromatography step with the column equilibrated with $20 \mathrm{mM}$ Tris. $\mathrm{HCl}, 200 \mathrm{mM}$ salt and 5mM DTT (Superdex 200 Increase 10/300, GE Healthcare).

\section{Immunoprecipitation and Competition Assays}

Inducible, stable cell lines expressing indicated YFP-tagged bait were lysed in a low salt lysis buffer (50 mM Tris-HCl pH 7.5, 50 mM NaCl, 1 mM EDTA, 1 mM DDT, 0.1\% NP-40, protease- and phosphatase inhibitors), unless otherwise stated. In some experiments, the same lysis buffer with $150 \mathrm{mM} \mathrm{NaCl}$ was used. Lysates were immunoprecipitated with GFP. trap beads (ChromoTek) according to the manufacturer's recommendation. The beads were washed three times with wash buffer $(50 \mathrm{mM}$ Tris- $\mathrm{HCl} \mathrm{pH} \mathrm{7.5,} 1 \mathrm{mg} / \mathrm{mL}$ BSA, $20 \%$ glycerol, 
and $1 \mathrm{mM}$ DTT) and eluted in 2x sample buffer. For the peptide competition assays, a peptide containing LxxIxE motif (LPRSSTLPTIIHEEEEELSLC) or a control mutant peptide that was unable to bind B56 (LPRSSTLPTA $\underline{A}$ AEEEELSLC) was used. For the competition assay with Sgo1 proteins, purified full-length hSgo1 or hSgo1 1-154 described above were used. The peptides/proteins were incubated with cell lysates 30 minutes prior to the addition of GFPtrap beads.

IP samples were resolved with 4-12\% Bis-Tris gels (Thermo Fischer Scientific), transferred to PVDF membranes. LI-COR Odyssey imaging system was used for visualization, and signals were quantified using Image Studio software (LI-COR).

\section{Chromosome Spreads}

Indicated cells were seeded in a 6-well plate, RNAi knockdown was performed, and cells were synchronized using thymidine followed by nocodazole treatment. $48 \mathrm{~h}$ after RNAi transfection, mitotic cells were collected by shake-off. After hypotonic treatment with $\mathrm{KCl}$, cells were spun onto microscopy slides with a Shandon Cytospin centrifuge (Thermo Fischer), fixed with 4\% paraformaldehyde, and then immunocytochemistry was performed. Representative images were taken with a 100x objective on a DeltaVision fluorescent microscope under the same condition. The distance between the two peak intensities of CREST or YFP-CB was measured for 5 kinetochore pairs using imageJ and averaged for a single cell. At least 45 cells from minimum of 3 independent experiments were analyzed.

\section{Live Cell Imaging}

Live cell imaging was performed using a DeltaVision fluorescent microscope. Cells were seeded in a 8-well ibidi dish (ibidi) a day before filming, the media was changed to Leibovitz's L-15 (Life Technologies) immediately before the filming. Indicated channels were recorded at 7-8 minute intervals and data were analyzed using SoftWoRx (GE Healthcare). The time from nuclear envelope breakdown (NEBD) to anaphase was measured in single cells.

\section{Immunofluorescence}

Cells were seeded in a 6-well plate and treated as indicated. A day before fixing, cells were transferred in an 8-well ibidi dish. Cells were fixed in 4\% PFA for $10 \mathrm{~min}$ at room temperature, 
and standard immunocytochemical methods were used. Fluorescent microscopy was performed using a DeltaVision fluorescent microscope. To ensure quantitative image quality, the imaging parameters were kept constant for a given experiment.

\section{LacO-Lacl assay}

Sgo1-Lacl-GFP constructs were cloned in a pAceBac1-CMV background (Hadders et al, 2020). Bacmids were generated using the Bac-to-Bac system in conjunction with EMBacY cells (Berger et al, 2004; Bieniossek et al, 2012). Baculovirus was then produced by transfection of bacmids into Sf9 cells using standard procedures. P2 viruses were harvested after 5 days, filtered $(0.2 \mathrm{um})$ and stored at $4^{\circ} \mathrm{C}$ till use. The lacO-Lacl assays were performed as previously described in Hadders et al 2020. Briefly, U-2 OS LacO Haspin CM (CRISPR Mutant) cells were seeded on glass coverslips followed directly by addition of recombinant baculovirus encoding the Sgo1-Lacl-GFP variants or Lacl-GFP as a control. After 3-4 hours S-trityl-L-cysteine (STLC; $20 \mu \mathrm{M}$ ) was added overnight to block cells in mitosis. The next morning cells were fixed in 4\% PFA (v/v) in PHEM buffer $(60 \mathrm{mM}$ HEPES $\mathrm{KOH}, 20 \mathrm{mM}$ PIPES KOH, $\mathrm{pH}$ 6.8, $5 \mathrm{mM}$ EGTA, and $1 \mathrm{mM} \mathrm{MgCl}_{2}$ ) for 10-15 minutes followed by permeabilization in ice cold methanol for a minimum of 1 hour.

For immunofluorescence, cells were washed with PBS with 0.01\% Tween 20 (PBST), followed by blocking with $3 \%$ BSA in PBST for \pm 30 min. Cells were then incubated with primary antibodies diluted in 3\% BSA in PBST for $2 \mathrm{~h}$ followed by washing three times, again with PBST. Cells were then incubated with secondary antibodies, GFP-Booster and DAPI (500 ng/ul) in PBST + 3\% BSA for $1 \mathrm{~h}$. Coverslips were washed again, twice with PBST, followed by a final wash with PBS, before mounting onto glass slides using Prolong Diamond Antifade Mountant (Thermo Fisher Scientific).

Fluorescence images were acquired on a DeltaVision imaging system (GE Healthcare), upgraded with a seven-color InsightSSI Module \& TruLight Illumination System Module using a UPlanSApo 60×/1.40 objective and a CoolSnap HQ2 camera (Photometrics). 3D zstacks were collected and deconvolved using Softworx v6. Presented images are deconvolved maximum intensity projections. Quantifications were performed using an inhouse-developed macro in ImageJ that sets a threshold (Otsu) based on the GFP channel followed by measurement of all channels within this region of interest. 


\section{TurbolD proximity labeling and label-free LC-MS/MS analysis}

TurbolD proximity labelling assay was performed as described previously (Branon et al, 2018). Doxycycline-inducible TurbolD-Sgo1 WT, 3A and 4A stable cell lines were generated in HeLa cells, and $50 \mu \mathrm{M}$ biotin was added to the media $1 \mathrm{~h}$ prior to the harvest. Cells were collected by mitotic shake-off, lysed with RIPA buffer, and immunoprecipitation was performed using high capacity Streptavidin agarose beads (ThermoScientific). The beads were washed once with RIPA buffer, twice with $2 \%$ SDS, then again once with RIPA buffer and eluted in 2x sample buffer. Pull-downs were analyzed on a Q-Exactive Plus quadrupole or Fusion Orbitrap mass spectrometer (ThermoScientific) equipped with Easy-nLC 1000 (ThermoScientific) and nanospray source (ThermoScientific). Peptides were resuspended in 5\% methanol / $1 \%$ formic acid and analyzed as previously described (Kruse et al, 2020).

Raw data were searched using COMET (release version 2014.01) in high resolution mode (Eng et al, 2013) against a target-decoy (reversed) (Elias \& Gygi, 2007) version of the human proteome sequence database (UniProt; downloaded 2/2020, 40704 entries of forward and reverse protein sequences) with a precursor mass tolerance of +/- $1 \mathrm{Da}$ and a fragment ion mass tolerance of $0.02 \mathrm{Da}$, and requiring fully tryptic peptides $(\mathrm{K}, \mathrm{R}$; not preceding $P$ ) with up to three mis-cleavages. Static modifications included carbamidomethylcysteine and variable modifications included: oxidized methionine. Searches were filtered using orthogonal measures including mass measurement accuracy $(+/-3 \mathrm{ppm})$, Xcorr for charges from +2 through +4 , and $\mathrm{dCn}$ targeting a $<1 \%$ FDR at the peptide level. Quantification of LC-MS/MS spectra was performed using MassChroQ (Valot et al, 2011) and the iBAQ method (Schwanhausser et al, 2011). Missing values were imputed from a normal distribution in Perseus to enable statistical analysis and visualization by volcano plot (Tyanova et al, 2016). For further analysis, proteins had to be identified in the Sgo1 +biotin or Sgo1 WT samples with more than 1 total peptide and quantified in 2 or more replicates. Statistical analysis was carried out in Perseus by two-tailed Student's t-test. 
bioRxiv preprint doi: https://doi.org/10.1101/2020.12.18.423432; this version posted December 19,2020 . The copyright holder for this

preprint (which was not certified by peer review) is the author/funder, who has granted bioRxiv a license to display the preprint in

A

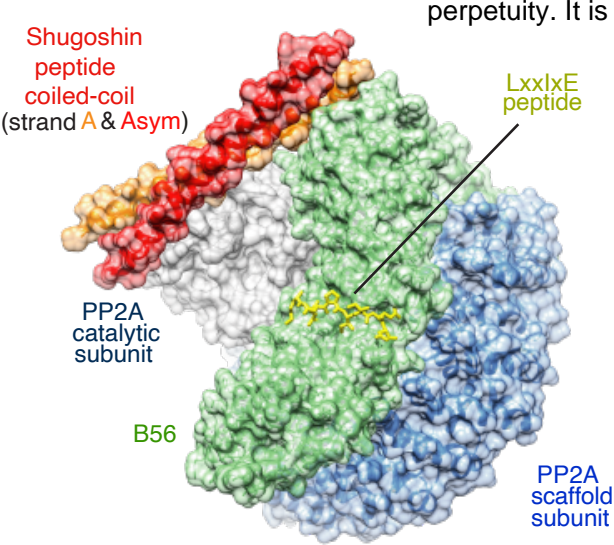

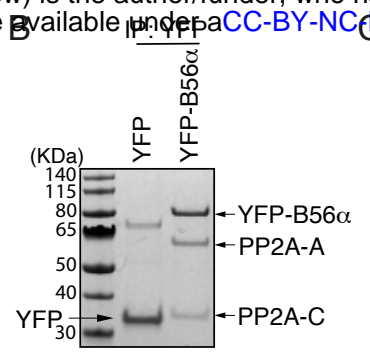

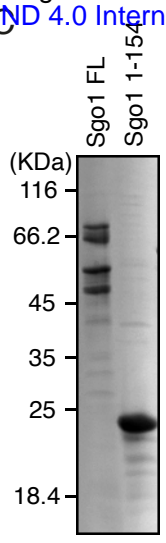

$+\frac{\stackrel{5}{5}}{\div}$

চ)
Input

य च

के

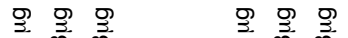

$+m^{-}$

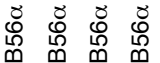

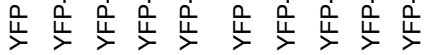

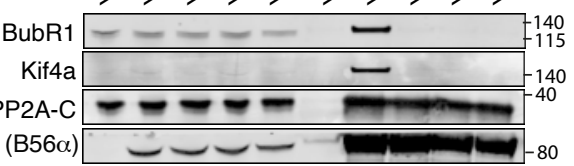

YFP (B56 $\alpha)$
$E$

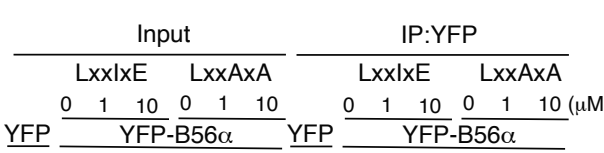

Sgo1 BubR1 - - - - - Kif4a PP2A-C YFP $(B 56 \alpha)$

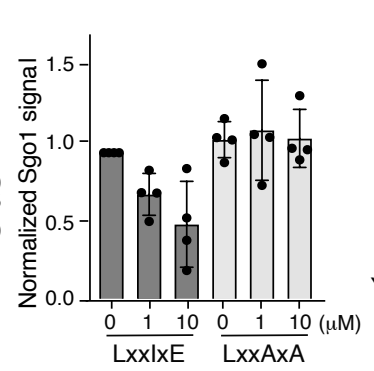

$\mathrm{F}$ Input

IP: YFP

YFP-B56 $\alpha$ variants YFP-B56 $\alpha$ variants

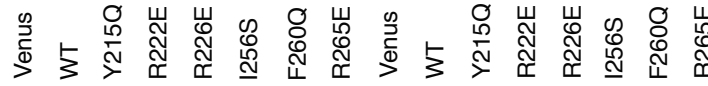

$$
\text { Sgo1 }
$$

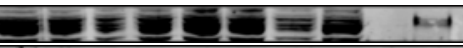

BubR1

PP2A-C YFP (B56 $\alpha)$

\section{$-65$


bioRxiv preprint doi: https://doi.org/10.1101/2020.12.18.423432; this version posted December 19, 2020. The copyright holder for this

preprint (which was not certified by peer review) is the author/funder, who has granted bioRxiv a license to display the preprint in

A Shugoshin $\begin{gathered}\text { Shugoshin } \\ \text { peptide } \\ \text { coiled-coil }\end{gathered}$
(Starian

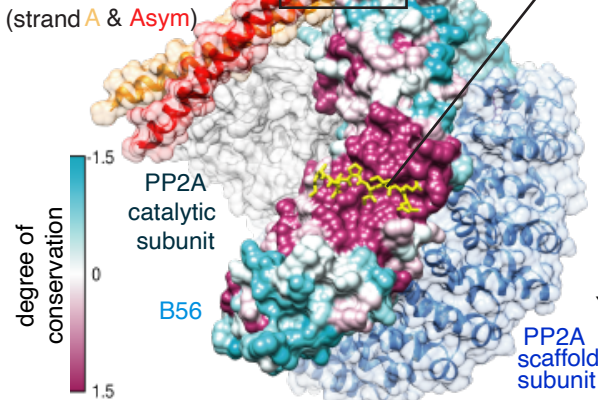
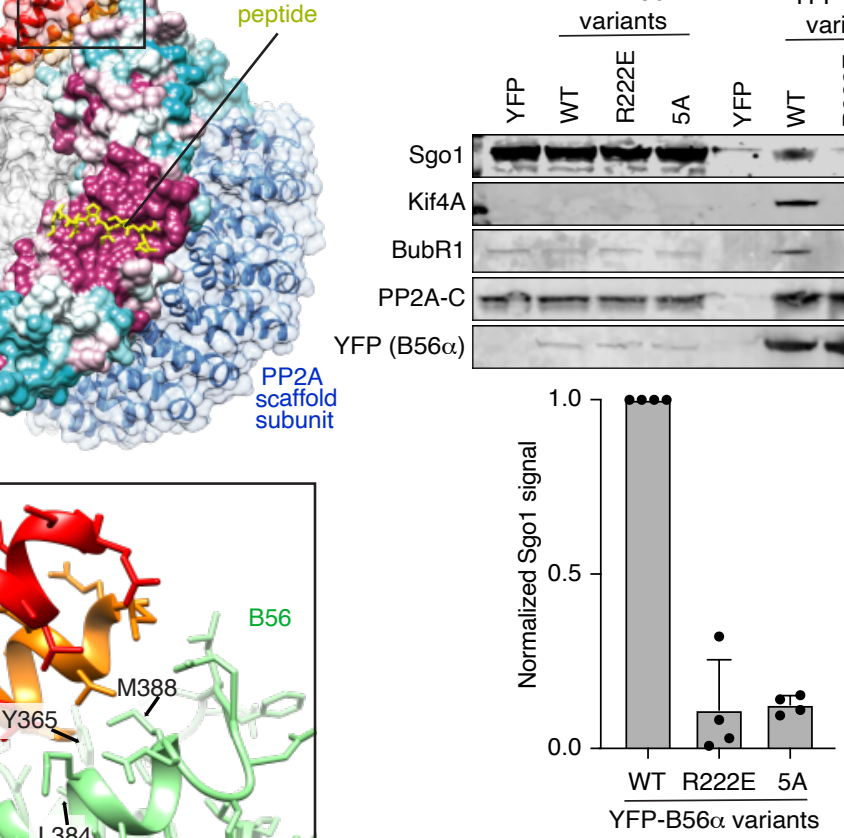

Input IP: YFP

YFP YFP-Sgo1 YFP YFP-Sgo1

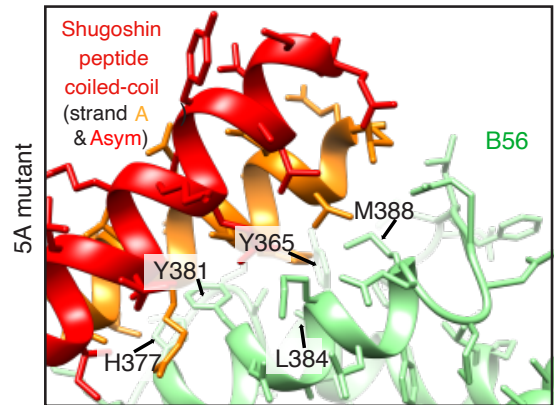

B56 RNAi
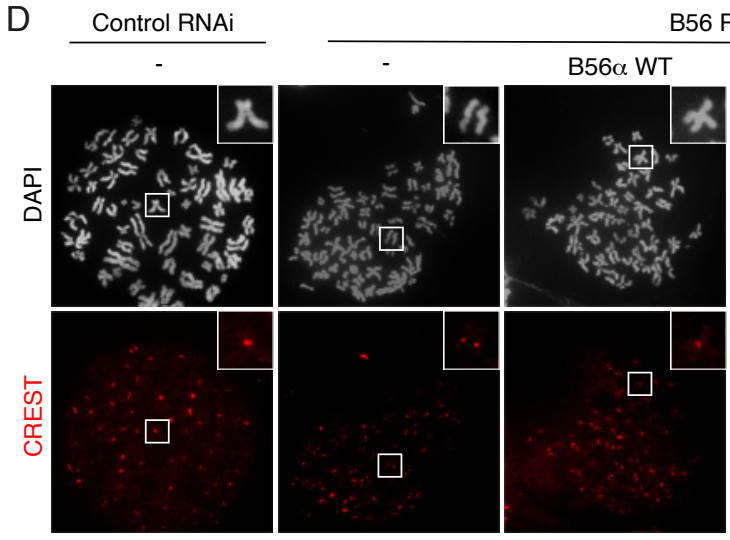

B56 $\alpha 5 \mathrm{~A}$

E
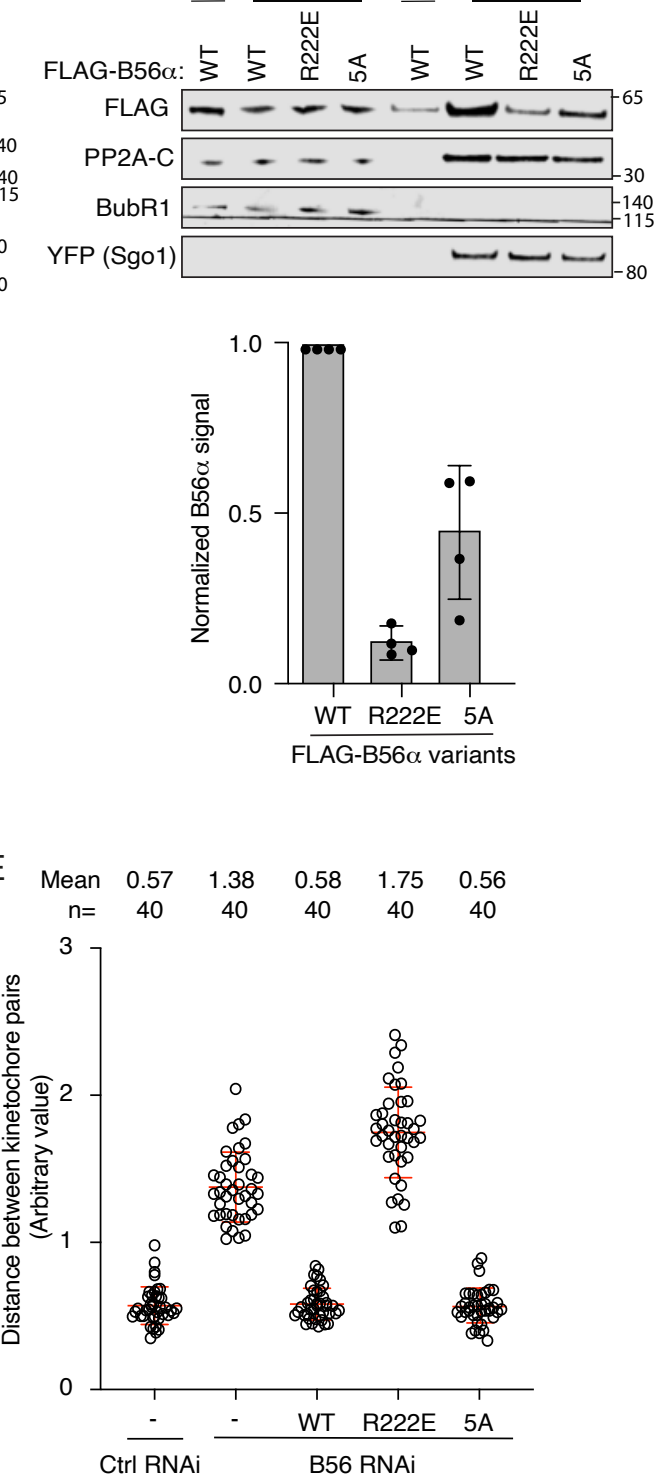

F Control RNAi

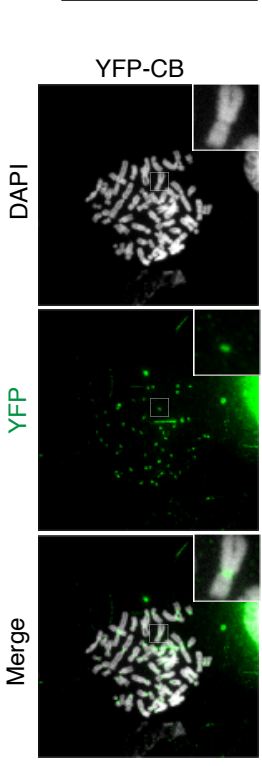

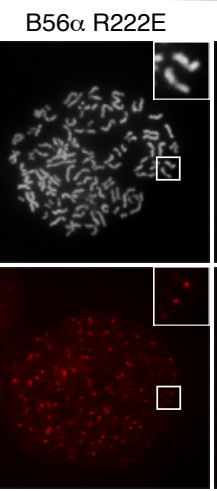

Sgo1 RNAi

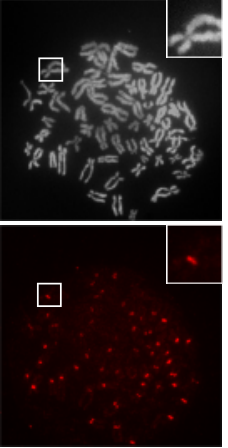

G

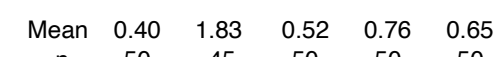

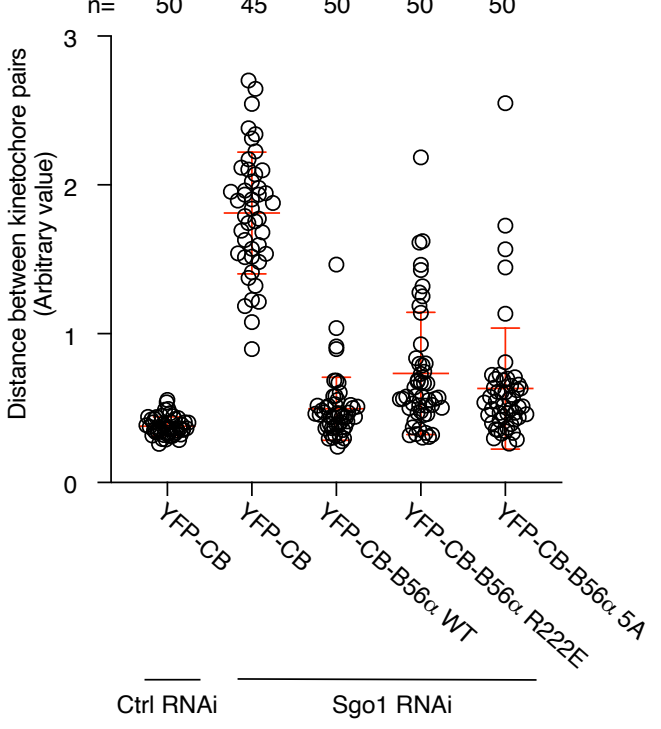


bioRxiv preprint doi: https://doi.org/10.1101/2020.12.18.423432; this version posted December 19, 2020. The copyright holder for this

preprint (which was not certified by peer review) is the author/funder, who has granted bioRxiv a license to display the preprint in
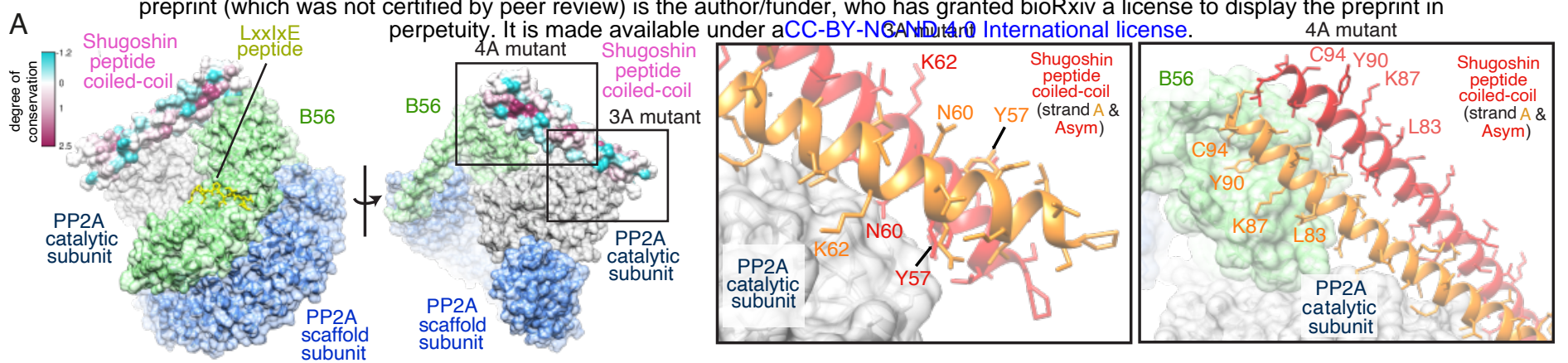

B
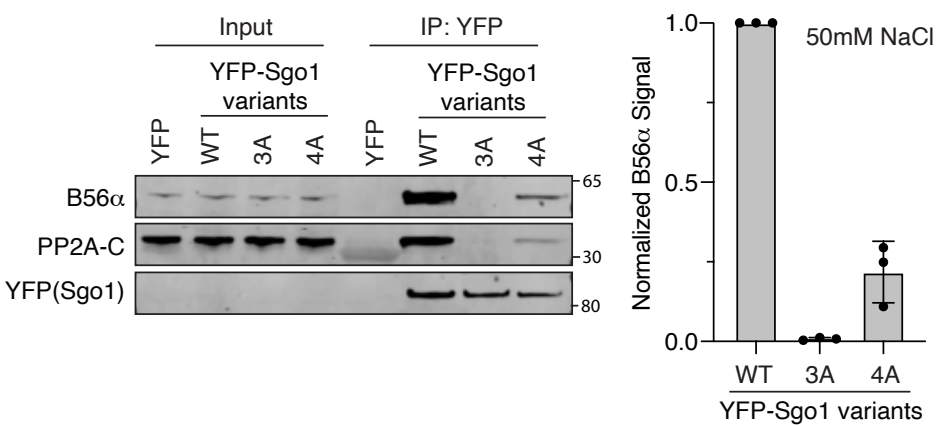

C

D
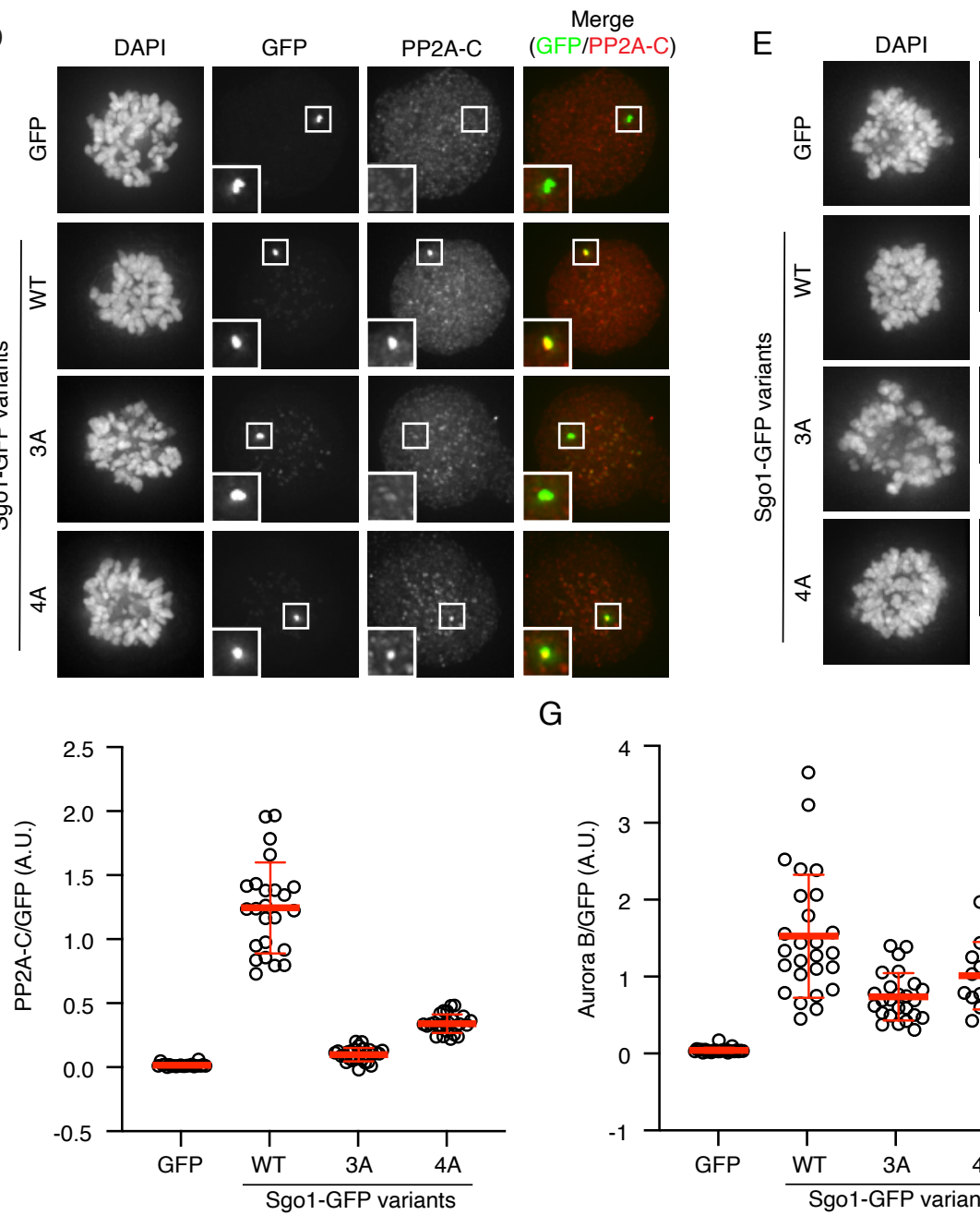

GFP

G

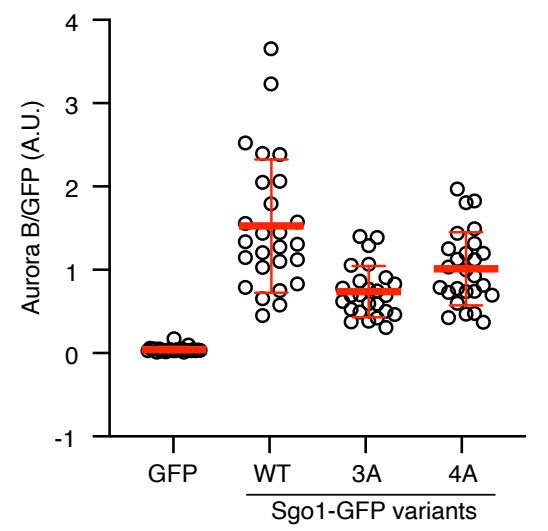

$\mathrm{H}$

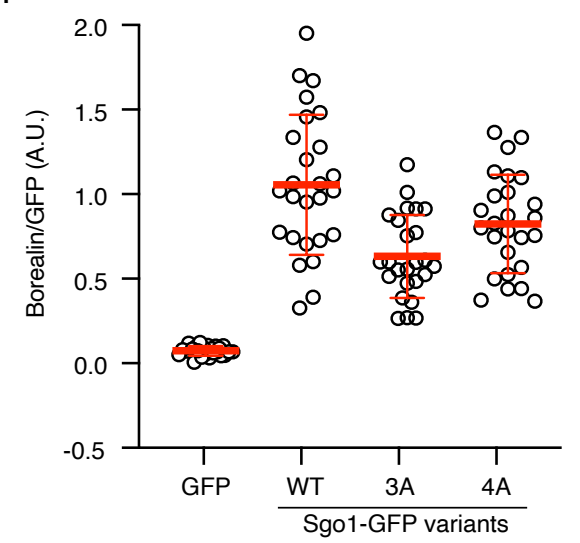

(

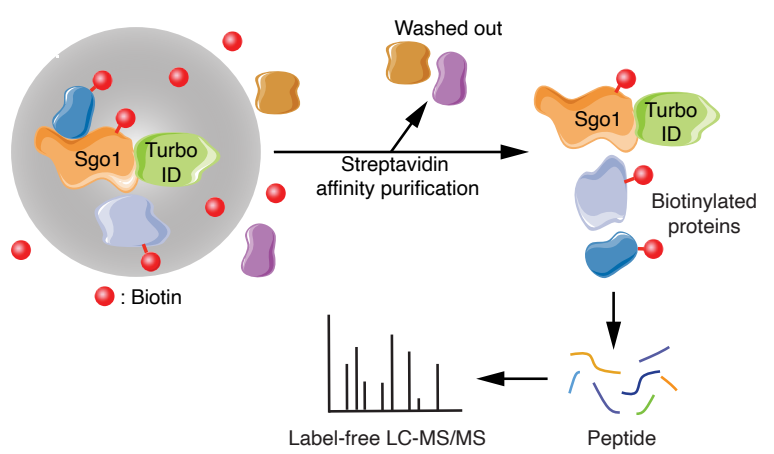

J

\begin{tabular}{|c|c|c|c|}
\hline & Log2 WT vs $3 A$ & Log2 WT vs $4 A$ & $\log 23 A$ vs $4 A$ \\
\hline B56 $\alpha$ & 3.41 & 4.41 & 0.99 \\
\hline B568 & 3.58 & 3.08 & -0.50 \\
\hline$B 56 \varepsilon$ & 2.93 & 3.36 & 0.43 \\
\hline B56 $\gamma$ & 2.34 & 1.11 & -1.23 \\
\hline Aurora B & 1.09 & 0.56 & -0.53 \\
\hline Borealin & 3.24 & $0.55(p=0.34)$ & -0.27 \\
\hline Incenp & $-0.26(p=0.1)$ & 0.57 & 0.83 \\
\hline
\end{tabular}


bioRxiv preprint doi: https://doi.org/10.1101/2020.12.18.423432; this version posted December 19, 2020. The copyright holder for this

preprint (which was not certified by peer review) is the author/funder, who has granted bioRxiv a license to display the preprint in

A perpetuity. It is made available under aCC-BY-NC-ND 4.0 International license.

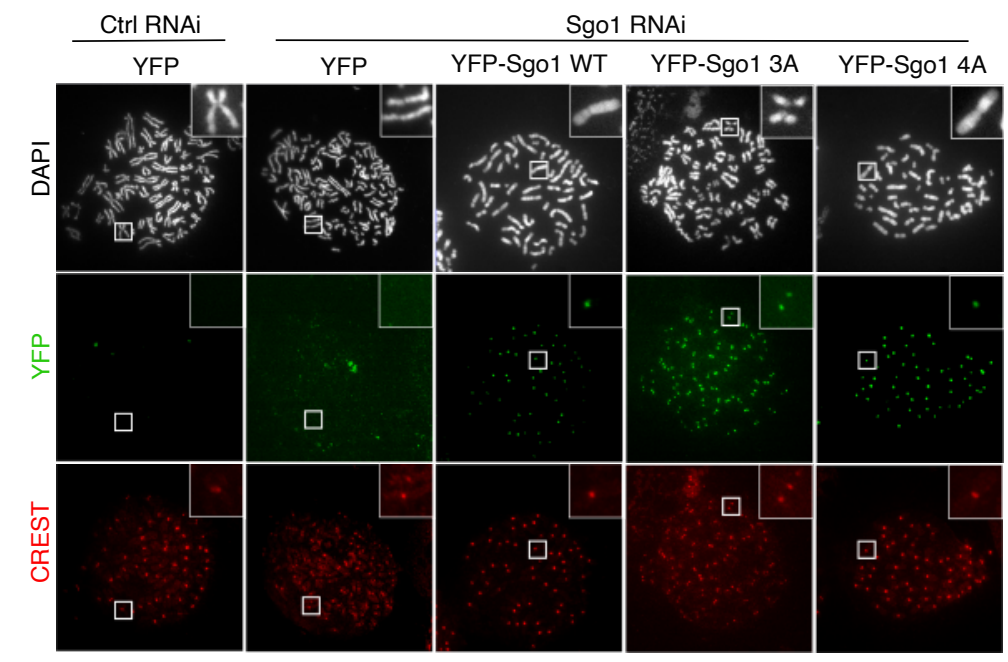

C
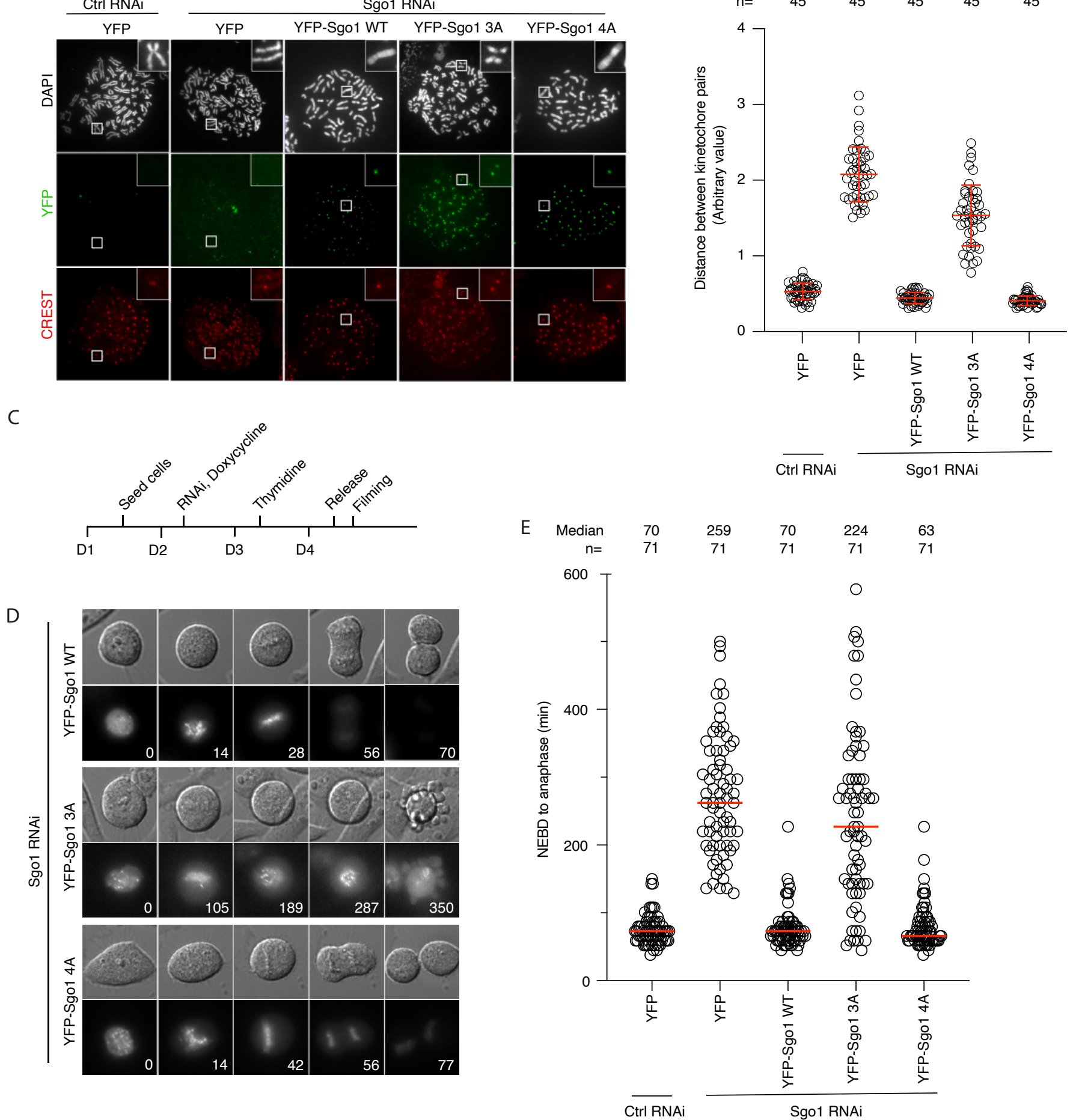
bioRxiv preprint doi: https://doi.org/10.1101/2020.12.18.423432; this version posted December 19, 2020. The copyright holder for this

preprint (which was not certified by peer review) is the author/funder, who has granted bioRxiv a license to display the preprint in perpetuity. It is made available under aCC-BY-NC-ND 4.0 International license.

A

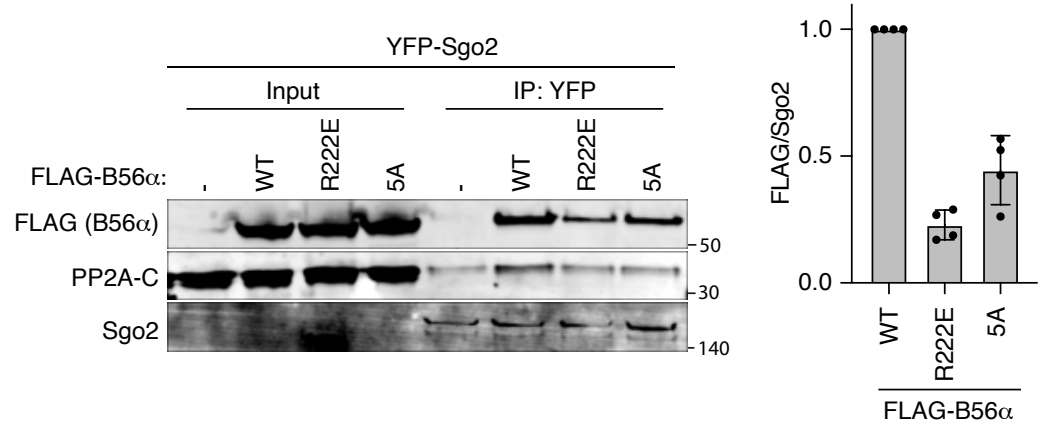

B

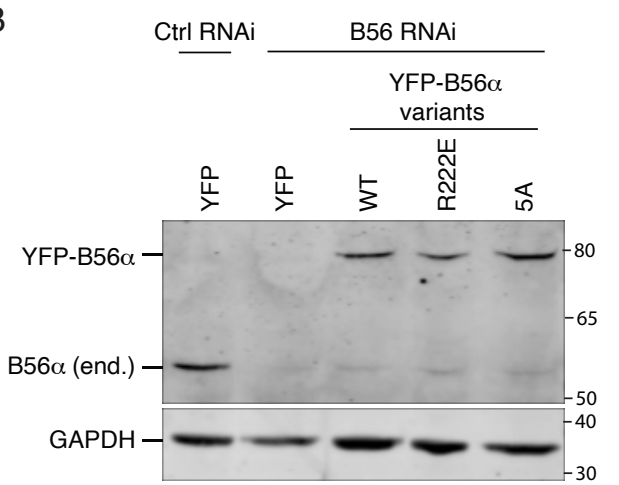

C

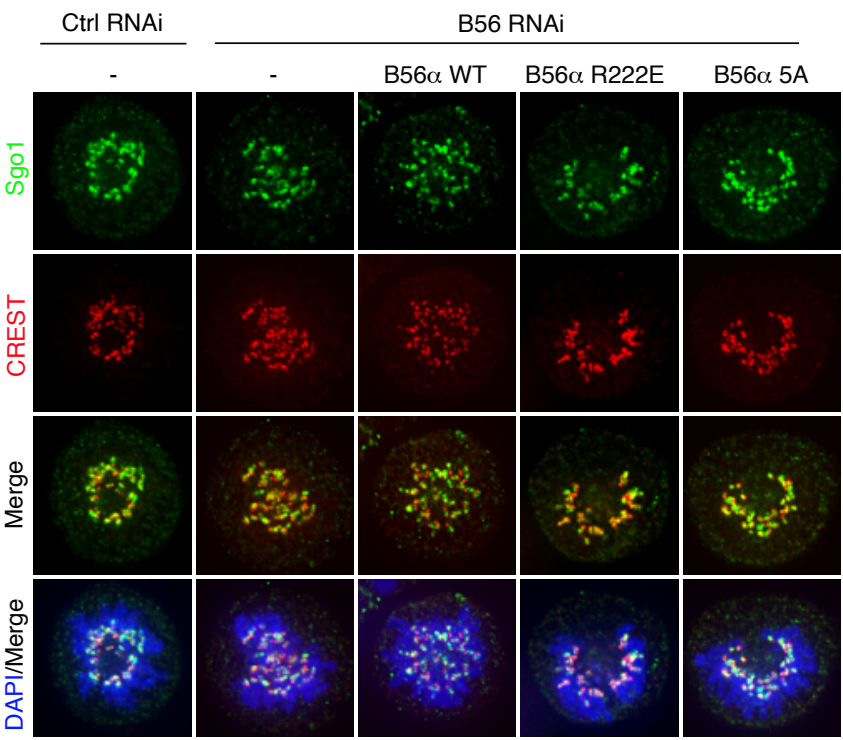

D

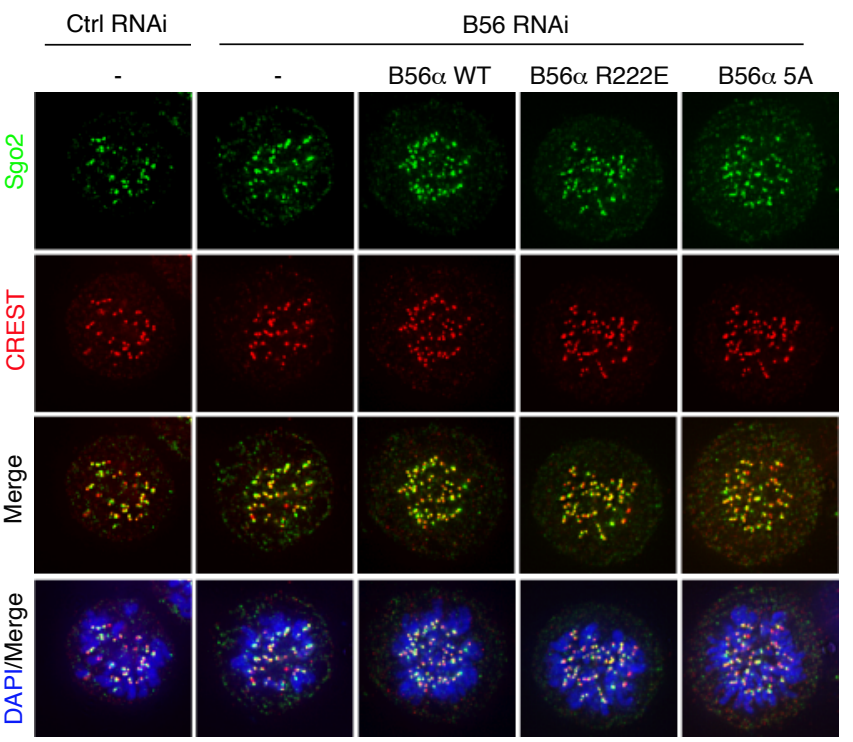

E

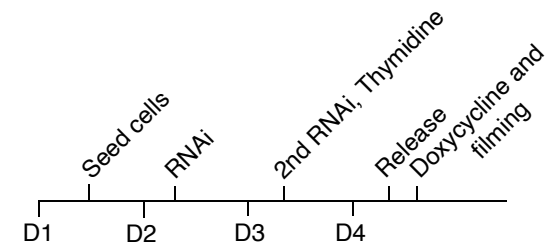

F
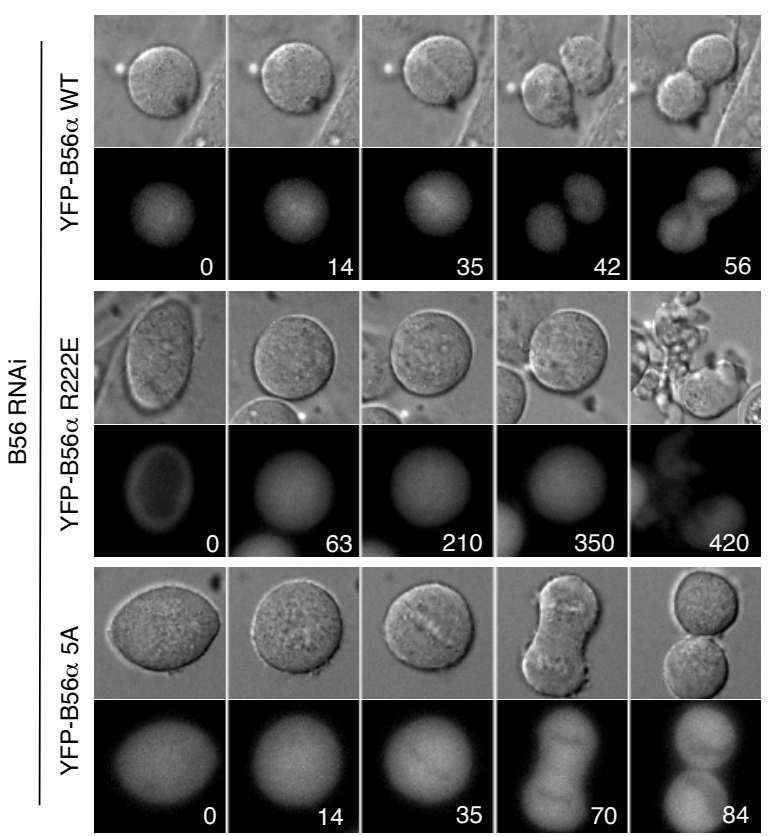

G

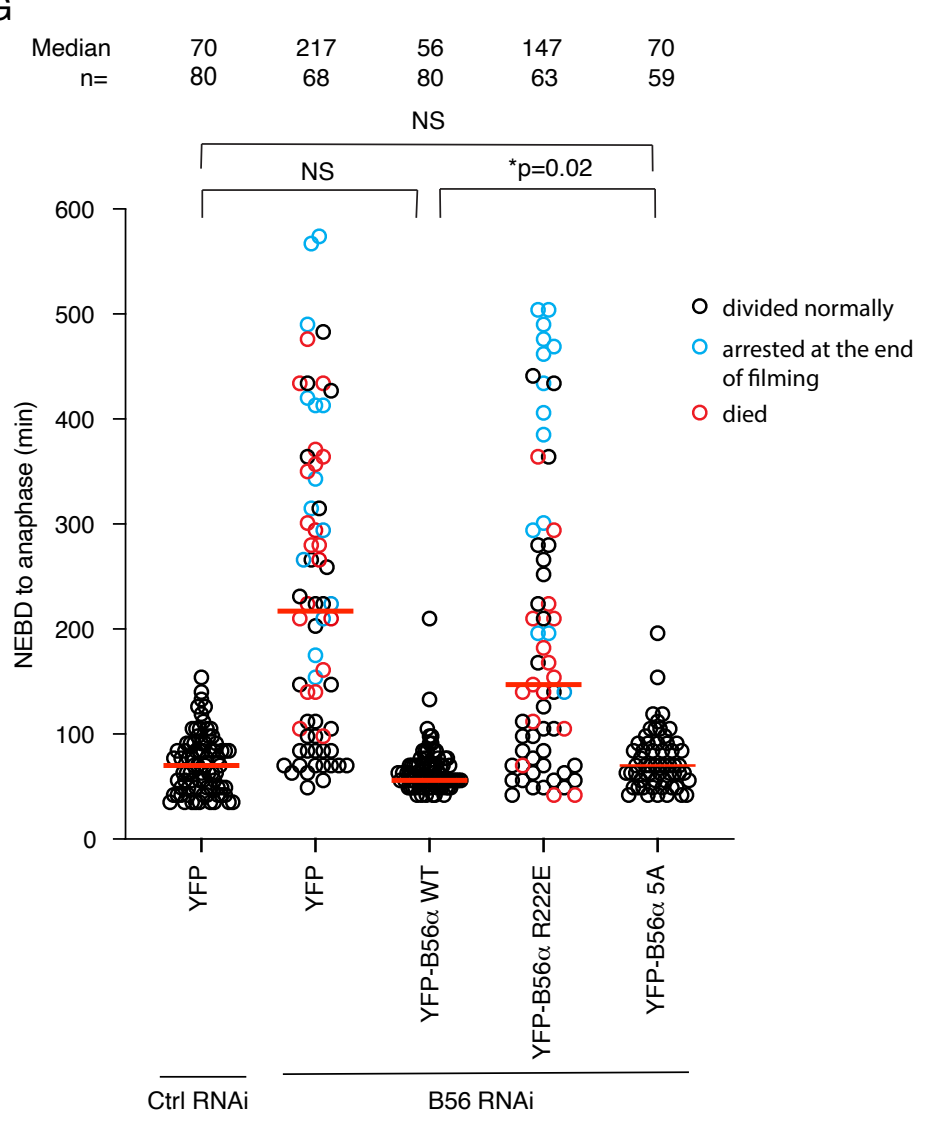


bioRxiv preprint doi: https://doi.org/10.1101/2020.12.18.423432; this version posted December 19, 2020. The copyright holder for this A preprint (which was not certified by peer review) is the author/funder, who has granted bioRxiv a license to display the preprint in Input perpetuityp:Itispmade available undB aCC-BY-NC-ND 4.0 International license.
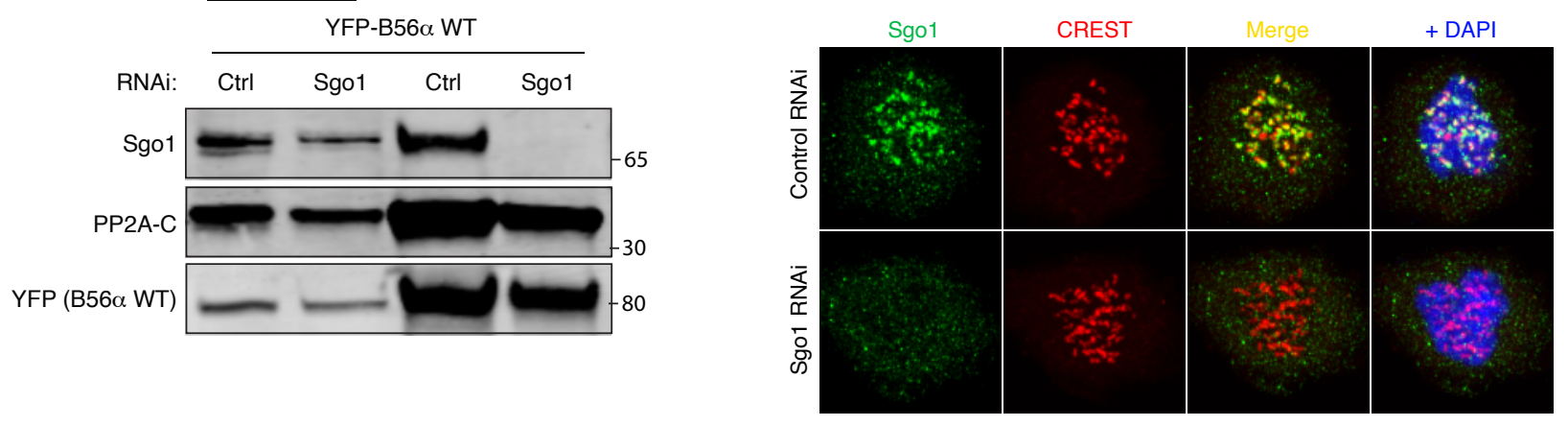

C

Human

Bird

47 ITNTSTLLKNY $3 \mathrm{~A}$

$4 \mathrm{~A}$

Reptile

Amphibian

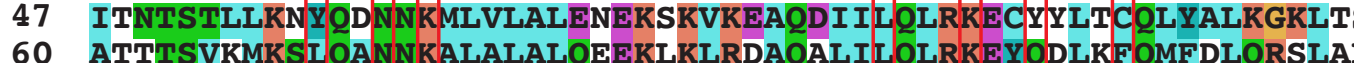

105

60 ATTTSVKMKSI QANNKALALALQEEKLKLRDAQALILQLRKEYQDLKFQMFDLQRSLAL 118

Fish

DNSSVQLKSF QENNRALALALEVEKGKVKDAQDI I HLKREYQHLKF QVMVLQRKLGL

224

44 AVVKPLVLKSVOANNKALAVALOAEREKVROAOGVI OLKKEROTLLFHLLMLKRTLRE

120 
bioRxiv preprint doi: https://doi.org/10.1101/2020.12.18.423432; this version posted December 19, 2020. The copyright holder for this

preprint (which was not certified by peer review) is the author/funder, who has granted bioRxiv a license to display the preprint in

A
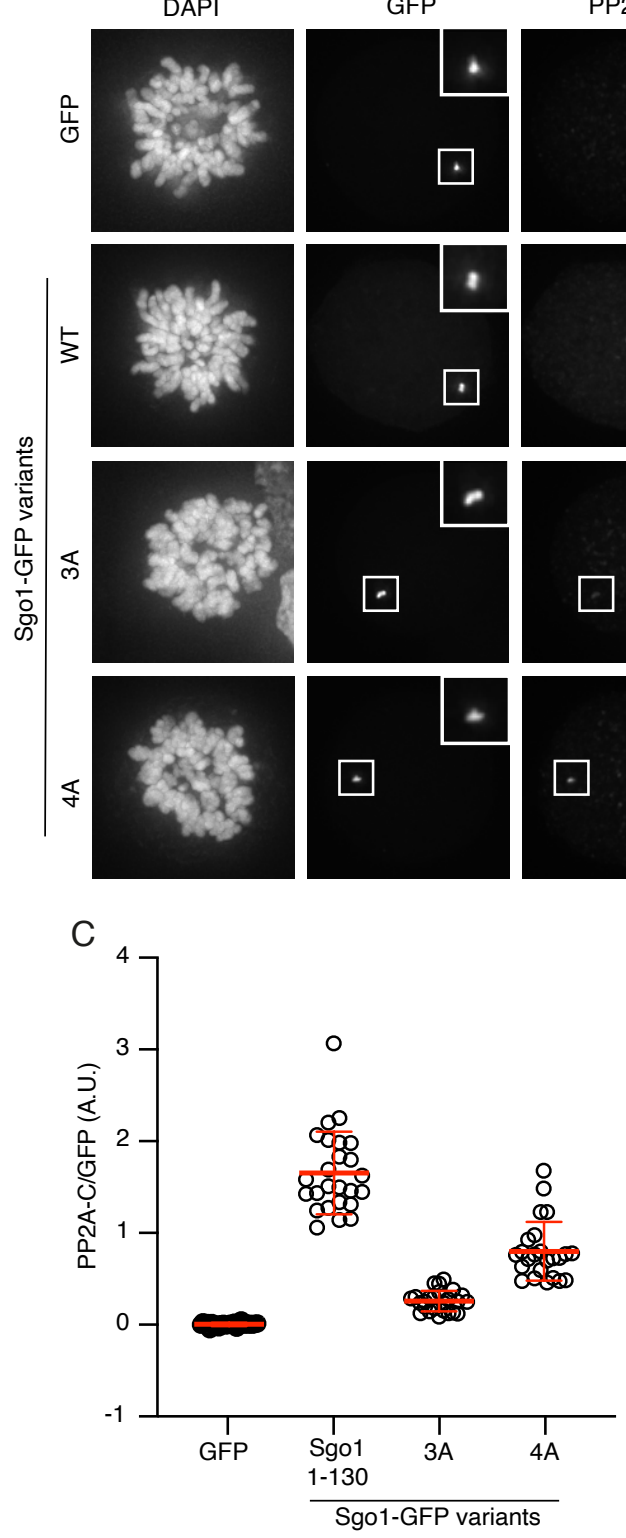

F \begin{tabular}{lllllllll} 
& \multicolumn{2}{c}{ Input } & & \multicolumn{1}{l}{ IP: Streptavidin } \\
Biotin & - & - & + & + & - & - & + & + \\
ycline & - & + & - & + & - & + & - & +
\end{tabular}

Sgo1 (TurbolD-Sgo1)

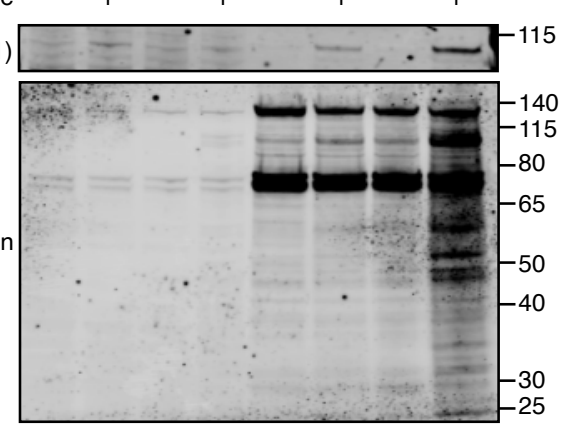

PP2A-C
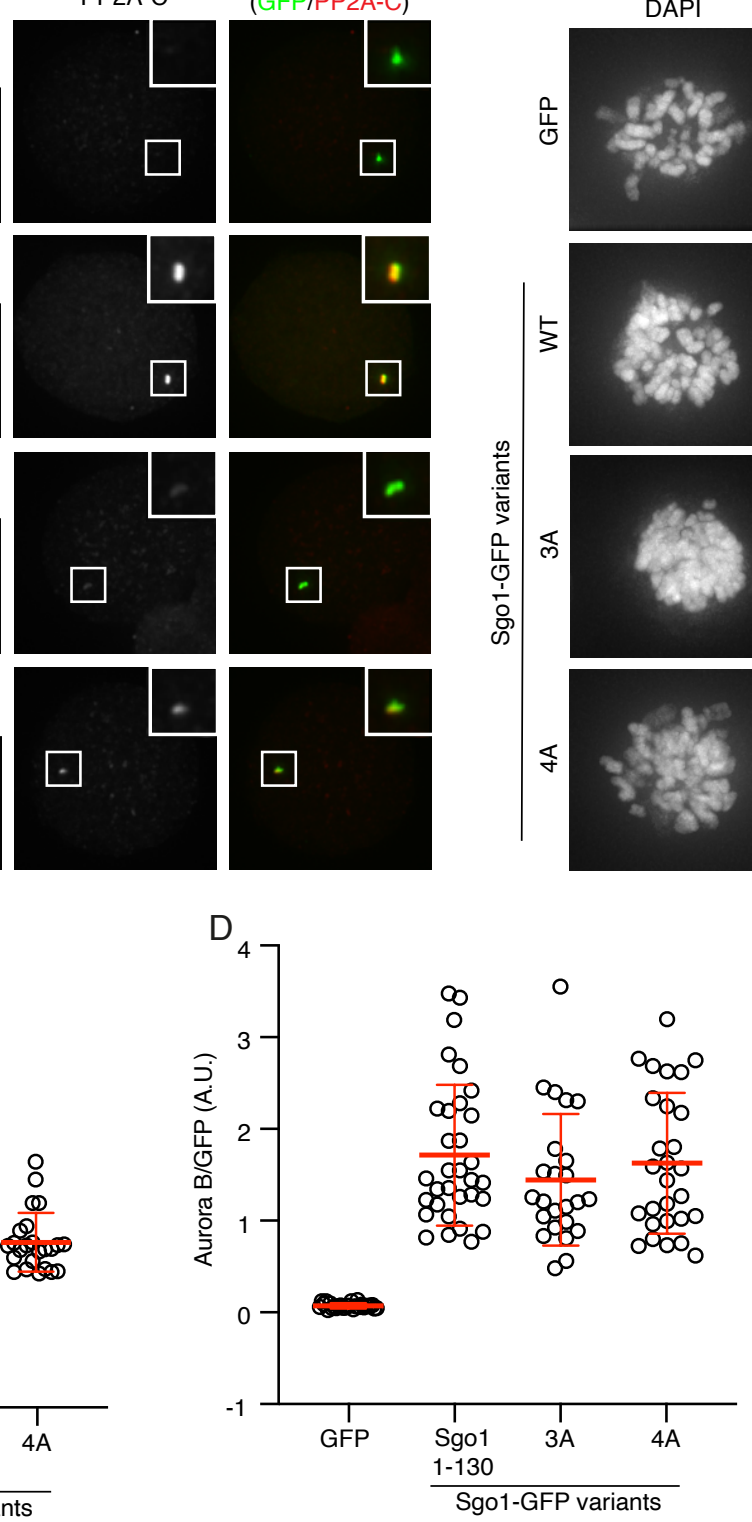

G
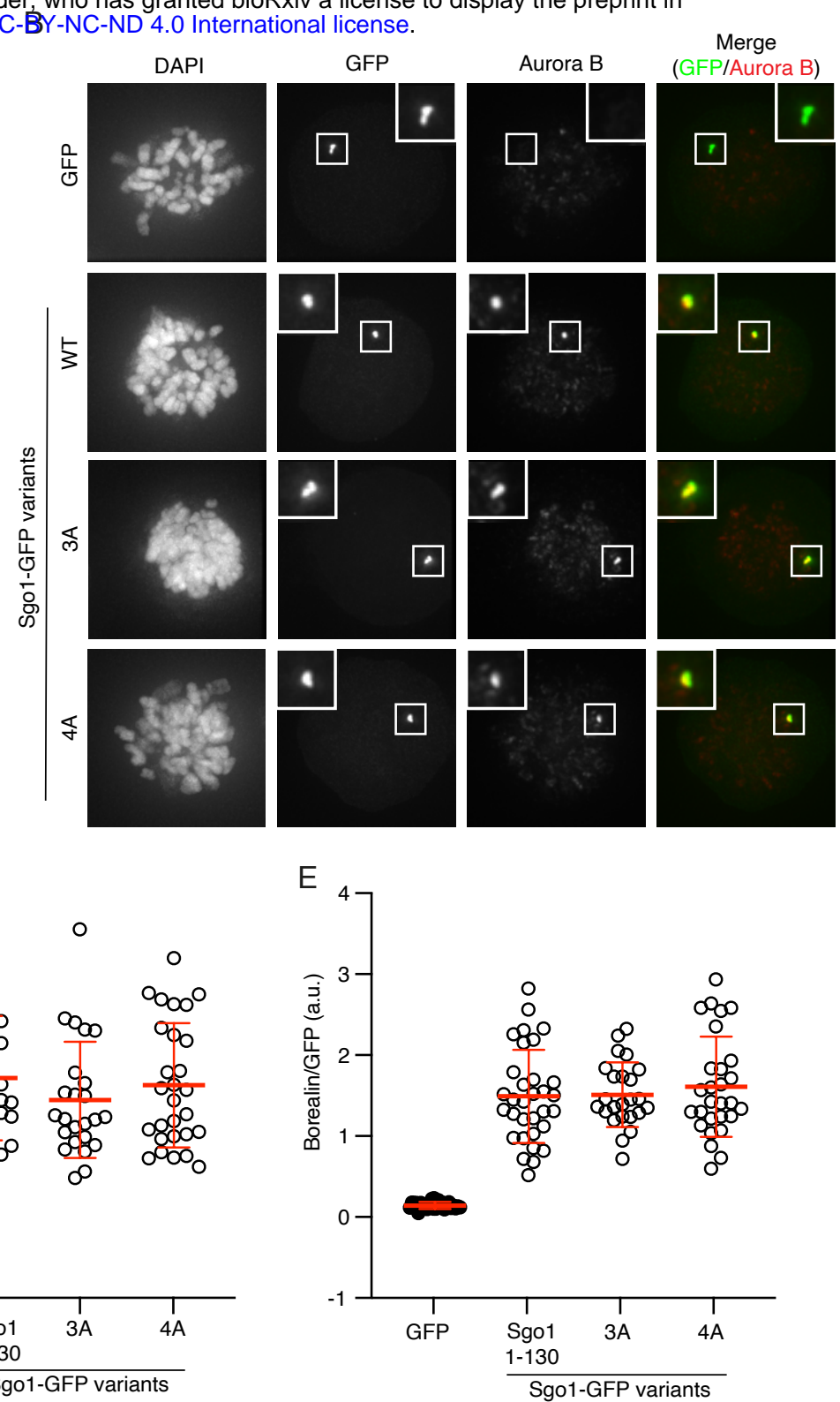

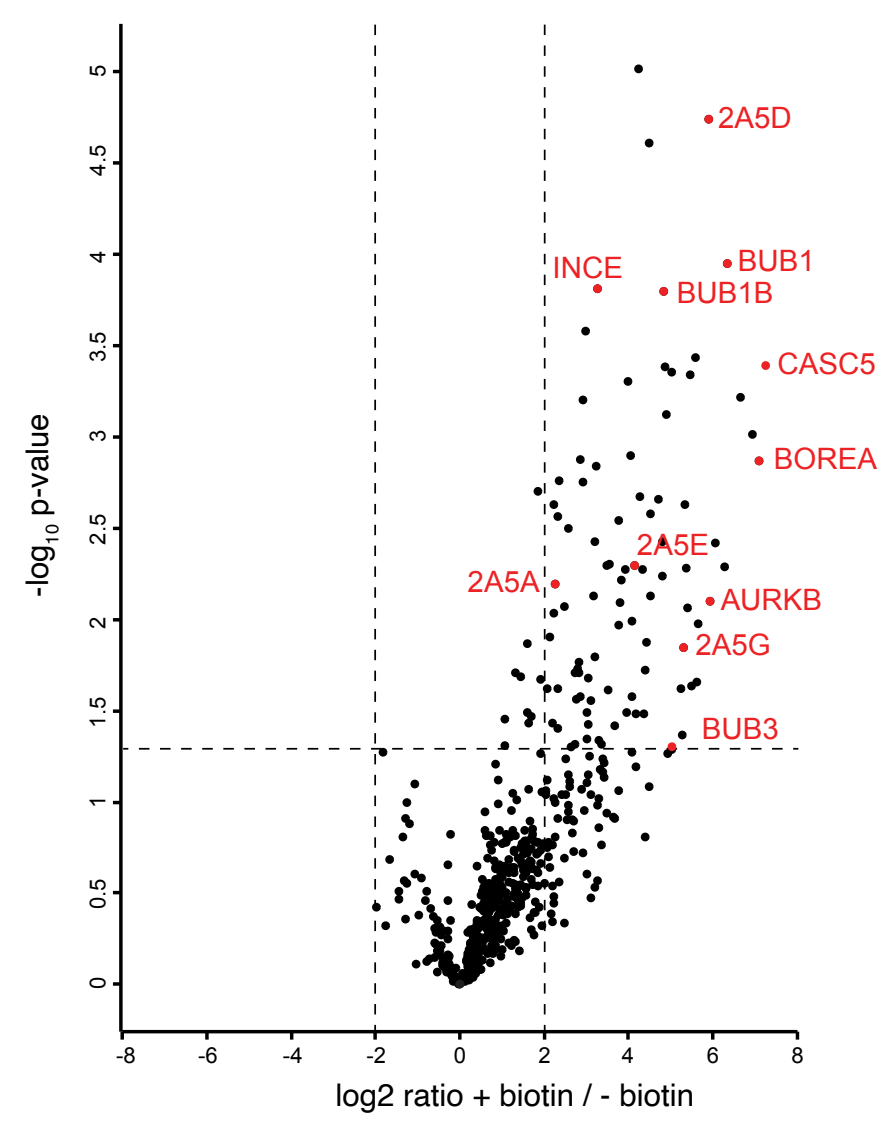

Supplemental Figure 3 
bioRxiv preprint doi: https://doi.org/10.1101/2020.12.18.423432; this version posted December 19, 2020. The copyright holder for this preprint (which was not certified by peer review) is the author/funder, who has granted bioRxiv a license to display the preprint in

A

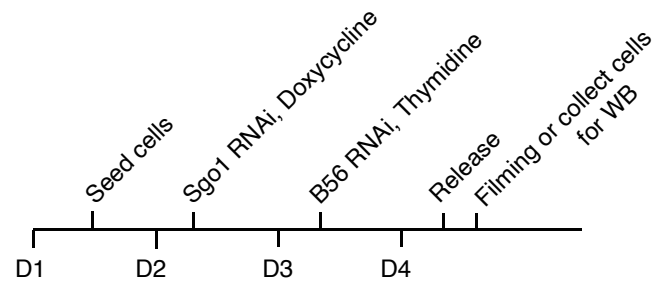

B

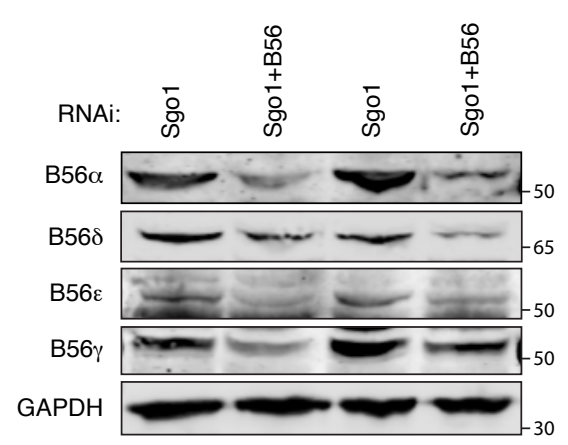

C

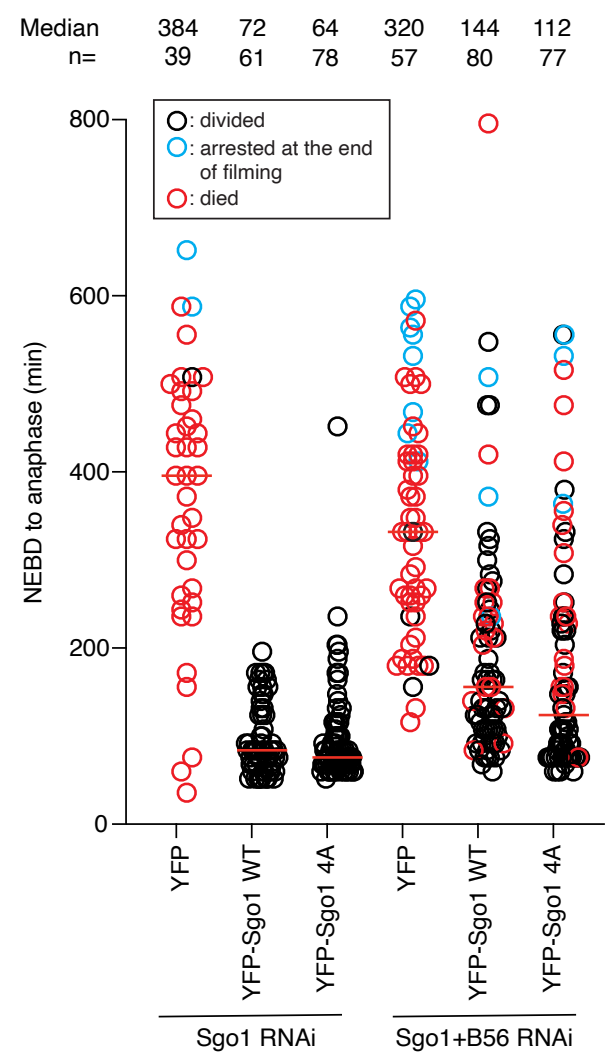

